\title{
Research Square \\ Decoding the olfactory map: targeted transcriptomics link olfactory receptors to glomeruli
}

Kevin Zhu ( $\nabla$ kevin.w.zhu@duke.edu )

Duke University

\section{Shawn Burton}

Lehigh University https://orcid.org/0000-0002-8907-6487

\section{Maira Nagai}

Duke University

\section{Justin Silverman}

Pennsylvania State University

\section{Claire De March}

Duke Medical Center

\section{Matt Wachowiak}

Boston University

Hiroaki Matsunami

Duke University Medical Center

\section{Article}

Keywords: olfactory map, glomeruli, olfactory receptors

Posted Date: November 11th, 2021

DOI: https://doi.org/10.21203/rs.3.rs-1009542/v1

License: (c) (1) This work is licensed under a Creative Commons Attribution 4.0 International License.

Read Full License

Version of Record: A version of this preprint was published at Nature Communications on September 1st, 2022. See the published version at https://doi.org/10.1038/s41467-022-32267-3. 


\section{TITLE:}

2 Decoding the olfactory map: targeted transcriptomics link olfactory receptors to glomeruli 3

4 AUTHORS:

5 Kevin W. Zhu ${ }^{1}$, Shawn D. Burton ${ }^{2,3}$, Maira H. Nagai ${ }^{1}$, Justin D. Silverman ${ }^{4,5,6,7}$, Claire A. de 6 March $^{1}$, Matt Wachowiak ${ }^{3,{ }^{*}}$, Hiroaki Matsunami ${ }^{1,8,9,{ }^{*}}$

8 AFFILIATIONS:

9 1Department of Molecular Genetics and Microbiology, Duke University Medical Center;

10 Durham, North Carolina, 27710, USA

11 'Department of Biological Sciences, Lehigh University; Bethlehem, Pennsylvania, 18015,

12 USA

13 'Department of Neurobiology, University of Utah School of Medicine; Salt Lake City, Utah, 14 84112, USA

$15{ }^{4}$ College of Information Science and Technology, Pennsylvania State University; University 16 Park, Pennsylvania, 16802, USA

17 5epartment of Statistics, Pennsylvania State University; University Park, Pennsylvania, 18 16802, USA

$19{ }^{6}$ Department of Medicine, Pennsylvania State University; Hershey, Pennsylvania, 17033, USA

$20 \quad{ }^{7}$ Institute for Computational and Data Science, Pennsylvania State University; University 21 Park, 16802, USA

$22{ }^{8}$ Department of Neurobiology, Duke University Medical Center; Durham, North Carolina, 2327710, USA

24 9Duke Institute for Brain Sciences, Duke University; Durham, North Carolina, 27710, USA 25 “Corresponding authors. Email: hiroaki.matsunami@duke.edu, matt.wachowiak@utah.edu 


\section{ONE SENTENCE SUMMARY:}

28 Targeted transcriptomics of olfactory receptor mRNA in olfactory bulb sections determines spatial positions for glomeruli.

ABSTRACT:

Sensory processing in olfactory systems is organized across olfactory bulb glomeruli, wherein axons of peripheral sensory neurons expressing the same olfactory receptor coterminate to transmit receptor-specific activity to central neurons. Understanding how receptors map to glomeruli is therefore critical to understanding olfaction. High-throughput spatial transcriptomics is a rapidly advancing field, but low-abundance olfactory receptor

expression within glomeruli has previously precluded high-throughput mapping of receptors to glomeruli. Here we combined sequential sectioning along the anteroposterior, dorsoventral, and mediolateral axes with target capture enrichment sequencing to overcome lowabundance target expression. This strategy allowed us to spatially map $86 \%$ of olfactory receptors across the olfactory bulb and uncover a relationship between OR sequence and glomerular position.

\section{MAIN TEXT:}

The organization of axonal projections from olfactory sensory neurons (OSNs) in the olfactory epithelium (OE) to glomeruli on the olfactory bulb $(\mathrm{OB})$ forms the olfactory map ${ }^{1-4}$. In the mouse, each canonical OSN expresses a single olfactory receptor (OR) or trace amineassociated receptor (TAAR) allele from a repertoire of over 1000 OR and TAAR genes. Insights into the organization of the olfactory map were first obtained using in situ hybridization, where OR transcript probes indicated the convergence of OSN axons into 
51 discrete structures called glomeruli on the OB surface, which range from 50 to $120 \mu \mathrm{m}$ in

52 diameter ${ }^{3,5,6}$. Later, this organization was more clearly visualized through the use of gene-

53 targeted mouse lines, which demonstrated that glomeruli are formed from the axonal

54 convergence of OSNs expressing the same OR gene ${ }^{7}$. Together these studies established

55 the convergence of homotypic OSN axons to stereotyped glomeruli whose positional variability ranges from 75 to $270 \mu \mathrm{m}$ depending on OR identity ${ }^{5,8}$. Because each glomerulus represents a single OR and a single odorant can bind multiple ORs, odor signals detected in the OE are transformed into a map of glomerular activity on the $\mathrm{OB}^{9-13}$.

To date, glomerular positions for less than $5 \%$ of mouse ORs are available, and further progress has been stymied due to the low-throughput, laborious, and time-consuming aspects of currently available methodologies for mapping each OR in the expansive murine repertoire ${ }^{7,8,13-29}$. Furthermore, the ability to compare locations between multiple glomeruli is limited among these studies due to the lack of reference landmarks on the OB and differences between methodologies. A more efficient approach for mapping OR axon projections to $\mathrm{OB}$ glomeruli would serve to generate a more comprehensive and informative map that would serve as a foundation for further functional studies of odor coding and processing. In this study, we demonstrate that target capture enrichment on sequential samples from the OB enables detection of low-abundance OR and TAAR mRNA in the axon termini of OSNs. Using this approach, we map $86 \%$ of the 1118 ORs and TAARs along the anteroposterior, mediolateral, and dorsoventral axes and combine these data to generate a three-dimensional model of glomerular positions with a precision of $141 \mu \mathrm{m}$. We examine the relationship between OR sequence and OB position, identify the set of ORs and TAARs expressed within dorsal glomeruli accessible to functional imaging, and generate genetargeted mouse lines for two dorsal glomerular ORs amenable to functional characterization 75 in vivo. 
RESULTS

\section{Targeted capture consistently enriches OR transcripts}

78

Previous studies have detected low levels of OR mRNA in OSN axon terminals, identifying the glomerular positions for specific ORs within histological sections ${ }^{5,23,27,30}$. To quantify OR and TAAR transcripts in the OB we first performed conventional RNA-Seq on whole-OB tissue from a mouse at postnatal day 21 , an age when olfactory glomeruli are fully developed and finalized in their stereotyped positions ${ }^{31,32}$. Quantification of 25.7 million reads identified $410 / 1118(36.7 \%)$ intact ORs at an average abundance of 0.06 transcripts per million (TPM) (median OR TPM = 0, 6/15 (40\%) TAARs with TPM above 0, mean TAAR TPM $=0.077$, median TAAR TPM = 0) (Fig. 1, B and C), confirming the low abundance of OR mRNA in OSN axon terminals ${ }^{30,33}$.

To enrich sampling for OR transcripts, we designed a target capture array against chemosensory receptor gene families primarily targeting ORs and TAARs ${ }^{34}$. We applied this target capture array to the previously sequenced OB library and identified 842 of the 1118 ORs (75.3\%) and 10 of the 15 TAARs (66.7\%) (Fig. 1, A to C). Following targeted capture, these ORs were present in a set of 27.7 million reads at an average abundance of 360.27 TPM (median = 106.74 TPM, mean TAAR TPM = 871.66, median TAAR TPM = 64.62) resulting in a mean fold enrichment of 6005X (mean TAAR fold enrichment $=11320 \mathrm{X})$ (Fig. 1 , B and C). Spearman's rho for OR and TAAR transcript abundances between uncaptured and captured samples was $0.71\left(P<2.2 \times 10^{-16}\right)$ (Fig. 1B). Further, four sets of independently captured technical replicates from two different OBs (two distinct spike-in mRNAs for RNA subsamples from each of the two bulbs with two technical replicates per subsample) exhibited a Spearman's rho of $0.95\left(P<2.2 \times 10^{-16}\right)$ (Fig. 1D and fig. S1D). The fold enrichment and correlation between pre- and post-capture samples indicates the targeted capture approach enriches the majority of ORs and TAARs in a highly consistent fashion as evidenced by the 
101 technical replicate correlation. The mean pairwise Spearman's rho for three biological

102 replicate OBs was $0.83\left(P<2.2 \times 10^{-16}\right)$, indicating the relative abundance of OR and TAAR

103 transcripts is conserved between individual animals (Fig. 1E and fig. S1E).

104 In summary, targeted capture consistently enriched OR and TAAR transcripts to levels

105 that facilitate positional analysis. This encouraged us to conduct targeted capture of ORs and

106 TAARs from sections of OB to determine which ORs were expressed in each section. We

107 sectioned from three directions, dorsoventral (DV), anteroposterior (AP), and mediolateral

108 (ML). We note that these three axes are not precisely orthogonal and not perfectly concordant

109 with the corresponding reference features such as DV zonal boundaries and medial surface

110 of the $\mathrm{OB}$.

112 Expression of ORs and TAARs in dorsoventral OB sections correlates with OE expression zones

114 Pioneering investigation established that ORs are expressed in overlapping,

115 continuous zones of the OE along the DV axis ${ }^{35,36}$. This zonal OE organization further

116 correlates with DV glomerular positions of $\mathrm{OR}$ expression in the $\mathrm{OB}^{7,23,37}$, with more recent

117 studies leveraging multiplexed assays and transcriptomics to map an expanded number of

118 ORs to more specific OE zones ${ }^{38,39}$. To comprehensively assess the relationship of OE-OB

119 DV zonal organization of OR expression, we collected $100 \mu \mathrm{m}$ sequential sections along the

120 OB DV axis for targeted transcriptomics to determine which ORs were expressed in each

121 section. Canonically, each OR is expressed in two glomeruli per bulb, both of which are

122 expected to be in similar positions along the DV axis. If enriched OR sequences are from

123 OSN axon terminals, we expect that each OR would be abundant in spatially clustered

124 sections which reflect the OE DV position from which the axons originate. 
After weighting and normalization across individual mice, the localization pattern of each OR and TAAR was limited to a single spatial cluster in a series of neighboring sections

127 for a majority of the capture-enriched transcripts (Fig. 2, A, B, and C). Uniform Manifold Approximation and Projection (UMAP) ${ }^{40}$ visualization of data from the three DV replicate mice (22 sections per replicate) placed sequential sections from replicate animals in an ordered, non-clustered path, indicating that spatially related sections have similar transcriptional profiles (Fig. 2D). Replicate heatmaps were similar to each other (mean $\mathrm{RV}_{\mathrm{adj}}=0.1803$ ), which supports the stereotyped targeting of glomeruli to local domains ${ }^{41,42}$. To assess concordance of $\mathrm{OB}$ and $\mathrm{OE}$ positions along the DV axis, we constructed an expressionweighted mean DV position for each OR from the average of all three DV replicate mice, which we found correlated with the published OE DV positions of each OR (Spearman's rho of mean position and OE index $=0.775, P<2.2 \times 10^{-16}$, Spearman's rho $=-0.690, P<2.2 \times$

$\left.1371^{-16}\right)(\text { Fig. 2E and fig. S2A })^{38,39}$. Along the DV axis, we found dorsal OE ORs, Class I ORs, and TAARs primarily located in the dorsal OB sections, while Class II ORs were distributed evenly along the DV axis, in agreement with previous mapping studies (dorsal vs ventral $P=$ $4.292 \times 10^{-95}$, Class I vs Class II $P=5.912 \times 10^{-20}$, TAAR vs OR $P=0.0001$, Mann-Whitney Utest) (Fig. 2F) ${ }^{29}$.

Anteroposterior spatial sections reflect stereotyped targeting of mirror-symmetric glomeruli

Using the same approach as for the DV axis, we examined $100 \mu \mathrm{m}$ sections along the AP axis of the OB to further assess the precision and reproducibility of our method and the stereotypical patterning of OR glomeruli. Prior studies have shown that each OR typically has two glomeruli located in distinct, yet spatially linked AP and mediolateral (ML) positions, as each $\mathrm{OB}$ is organized into half bulbs along a non-orthogonal symmetry line ${ }^{8,43,44}$. This 
symmetry typically leads to more posterior positioning of the medial glomerulus for an OR.

151 However, in cases where the target location of an OR is close to the symmetry line, both

152 glomeruli may appear in the same AP plane or only a single glomerulus may form ${ }^{45}$. Based

153 on these studies, we hypothesized that a majority of ORs would exhibit a bimodal expression

154 pattern along the AP axis.

155 Spatial expression patterns for 966 ORs and 14 TAARs, measured by the normalized

156 transcript abundances, were consistent across replicates with stereotyped AP glomerular

157 positions across $\mathrm{OBs}^{8,46}$. When sorted by position of mean expression, ORs primarily

158 displayed two peaks of expression, consistent with published studies for labeled ORs

159 displaying the medial glomerulus in a more posterior location relative to the lateral glomerulus

160 (Fig. 3, A and B, and fig. S3A $)^{8}$. Compared to the distribution of normalized OR expression

161 across the DV axis, ORs along the AP axis were distributed bimodally (Fig. 3C). Similar to the

162 DV axis, UMAP projections of gene expression values from the six AP replicate mice (23

163 sections per replicate) revealed correlated expression patterns and AP positions across each

164 OB (Fig. 3D). Across the AP axis, we found Class I ORs biased to the anterior set of sections,

165 while TAARs tended to be localized to the central portion of the axis (Class I vs Class II $P=$

$1662.017 \times 10^{-26}$, TAAR vs OR $P=0.7882$, Mann-Whitney U-test) (Fig. 3E), consistent with

167 previous studies examining glomeruli labeled in gene-targeted mice ${ }^{29,47}$. We also examined

168 our data for concordance against the set of 32 ORs cloned from the anterior, middle, and

169 posterior sections of an OB from Nakashima et al. ${ }^{48}$. The ORs cloned from the anterior and

170 middle OB $(n=13)$ on average occupied a significantly more anterior position in our data than

171 ORs cloned from the posterior OB $(n=18)($ anterior + middle OB cloned ORs vs posterior OB

172 cloned ORs $P=0.001$, Mann-Whitney U-test) (Fig. 3F and fig. S4A). We further divided these

173 cloned ORs across dorsal OE $(n=11)$ and ventral OE $(n=20)$ zones and found that both sets

174 displayed concordance with our AP data, with ORs cloned from the anterior and middle OB 
positions having a lower AP mean position than ORs cloned from the posterior OB (dorsal

176 OE: anterior and middle OB cloned ORs vs posterior OB cloned ORs $P=0.1636$, ventral OE: anterior and middle OB cloned ORs vs posterior OB cloned ORs $P=0.0117$, Mann-Whitney U-test) (fig. S4, B and C). Our high-throughput mapping of OR positions along the OB AP axis not only displays concordance with the largest available dataset of AP positions, but expands the available information from 32 to 966 ORs.

\section{Relationship of OR sequence and glomerulus position}

Gene-targeting approaches have identified a couple of examples in which closely related ORs target to nearby ${ }^{49,50}$. Our dataset with a majority of ORs assigned to specific AP positions allowed us to systematically interrogate whether ORs with similar sequences exhibit similar glomerular positions by computing pairwise alignments for all ORs. To assess this relationship in all dimensions, we additionally generated a ML dataset (3 replicates, 22 sections per replicate) (fig. S5, A to E). Due to the combined presence and sequence diversity of Class I ORs, Class II ORs, and TAARs on the dorsal surface of the OB, we separated our analysis into three groups: 1) Class I dorsal OB ORs, 2) Class II dorsal OB ORs, and 3) Class

191 II ventral OB ORs. To assess OR similarity, we calculated pairwise protein sequence identities and pairwise distances between the mean expression position for ORs along each dimension and compared the results for OR pairs using percent identity cutoffs corresponding to the level of homology used to classify OR genes as belonging to a family (40\%) (fig. S6A) ${ }^{51}$. When comparing OR pairs above and below the family-level threshold, we found both Class II dorsal and ventral ORs above the family level threshold displayed significant lower mean interglomerular distances across the AP, DV, and ML axes, suggesting topological relationship between the glomerular positions and family-level OR similarities for Class II ORs (Fig. 4A). In 
and mean expression position along the DV and ML axes (Fig. 4A and Table S6). We further examined this relationship by comparing ORs using the 60\% (subfamily level ORs) and $80 \%$ (highly similar) protein sequence identity thresholds ${ }^{51,52}$. Comparisons between family (40$60 \%$ ), subfamily (60-80\%), and highly similar ORs (>80\%) revealed that both dorsal and ventral Class II OR glomerular positions along all axes typically become more similar or do 205 not change with increasing sequence similarity (Fig. 4B). Class I ORs as a whole displayed largely non-significant differences in position except for comparisons against highly similar with a model in which overall similarities of ORs influence the relative glomerular locations.

We next sought to determine if any specific OR amino acid residues correlated with AP position. Due to the different relationships between sequence similarity and OB position for dorsal and ventral ORs, we examined Class II ORs using different cutoffs for the sets of the most anterior and most posterior ORs included in the analysis $(20 \%, 27.5 \%, 35 \%)$ to identify amino acid residues correlating with AP position (Fig. 4C and fig. S7A, B, C). We identified 22 residues whose physicochemical properties differed from all ventral Class II ORs (Fig. 4D and fig. S8C and S9) ${ }^{53}$. Notably, four consecutive residues that correlated with AP position were in the third intracellular loop, which has been shown to interact directly with the G protein during Class A GPCR activation (Fig. 4, D to F, and fig S8, A and B) ${ }^{54}$. Additionally, the phenylalanine within the KAFSTCXSH motif is sandwiched between four residues involved in G-protein binding ${ }^{55-57}$. These findings indicate residues that are at or near the sites of G-protein interactions are critical in determining glomerular position, which is consistent with the hypothesis that ligand-independent basal activity of ORs influences glomerular targeting ${ }^{45,48,58-60}$. 
To date, a systematic 3D model of OR glomerular positions has yet to be made for a

majority of the OR repertoire, but our high-throughput sequencing of spatial sections along the 3 cardinal axes may be combined to achieve such a result. UMAP plots of the mean position of ORs in each dimensional replicate indicated that ORs that are clustered on the OB surface are also clustered within the UMAP positions (Fig. 5A and fig. S10, A and B).

To account for the location of OR glomeruli on the outer surface of the OB, we extracted coordinates from a diffusion tensor imaging (DTI) model of the mouse $\mathrm{OB}$ to represent the approximate glomerular layer that would have been sampled by each section along each dimension of our targeted transcriptomics data ${ }^{61}$. We based our 3D reconstruction in a statistical approach to integrate our orthogonal sequencing data to determine which voxels on the OB surface where the probability for a given OR is highest ${ }^{62,63}$. We then developed an algorithm for the systematic reduction of positional probabilities across the whole OB surface into predicted OR glomerular positions. Lastly, we filtered out predictions with posterior median values below 0.0005 to account for ORs with low expression. Posterior median summaries for the resulting set of 709 ORs and TAARs predicted glomeruli positions as well as the raw positional probabilities for all 980 ORs and TAARs in all voxels are freely viewable as interactive 3D maps at kanazian.shinyapps.io/obmap/.

We assessed our algorithm by comparing predictions for Class I and II ORs, dorsal and ventral OE ORs, and the set of ORs examined via transgenic mouse lines by Zapiec et al. ${ }^{8}$. Predicted glomerular positions for Class I ORs, Class II ORs, and dorsal and ventral ORs were consistent with expectations based on OE zone, OR class, and our single-dimension data (dorsal vs ventral $P=9.188 \times 10^{-98}$, Class I vs Class $\| P=5.96 \times 10^{-14}$, TAAR vs OR $P=$ $2.682 \times 10^{-6}$, functional imaging surface enriched vs not-enriched $P=1.75 \times 10^{-24}$ (see below), Mann-Whitney U-test) (Fig. 5, B, C and F and fig. S11, B and C). Additionally, the distribution of predictions for the sets of Class I ORs and TAARs matched previously 
250 published findings for target domains (Fig. 5C and fig. 11B). Our current model predicts

251 glomerular positions for 700 ORs and 9 TAARs, with predicted positions outperforming

252 randomly selected ORs from the same DV zone and medial/lateral side for the subset of ORs

253 with known positions with a median error of $141 \mu \mathrm{m}$ (Fig. 5, D and E) $)^{8}$. The relative positions

254 of the Olfr1377 and Olfr881 glomeruli in the gene-targeted mouse lines (see below) were also

255 consistent with those predicted from the spatial transcriptomic data (Fig. 5D and figs. S11D,

256 S12D, S13 and S14), providing further support for our three-dimensional glomerular

257 predictions based on the spatial transcriptomic data. In summary, we found our three-

258 dimensional reconstruction of OR glomerular positions to be both in agreement with

259 established OR spatial features and to show greater concordance with these established

260 features than the sets of single-dimension target capture sequencing data alone. Collectively,

261 our results thus provide the first large-scale, unified, and systematic database of OR

262 glomerular positions for the mouse OB.

Identification of ORs within the dorsal functional imaging window

We sought to validate and extend our findings by examining specific ORs that map to

glomeruli on the dorsal-central OB surface, which has been extensively characterized by

267 functional imaging in vivo $9,11,12,46,64$. To define the set of ORs accessible under standard

268 functional imaging approaches, we collected tissue samples from OBs from C57BL6 mice (2

269 male, 2 female). Each OB was dissected into two parts, one containing the approximate

270 dorsal-central imaging area and the other containing the remainder of the OB (fig. S12A).

271 These 16 samples were processed for target capture sequencing and differential expression

272 analysis to identify ORs enriched in the functional imaging area.

273 A total of 121 ORs, including 27 Class I ORs and 94 Class II ORs, were consistently

274 enriched in the imaging surface (FDR $\leq 0.05$ and LogFC $>0$ ) (Fig. 5B, Fig. S12, B, E and F), 
with $96 \%$ of these ORs known to localize in dorsal OE zones ${ }^{38}$. We also found nine of the 15

TAARs enriched in the imaging surface, with no TAARs enriched in the remaining OB tissue

(fig. S12G), consistent with previous functional imaging of some TAAR glomeruli ${ }^{47}$. To

anatomically and functionally validate our expression analysis, we chose two ORs as targets

for the generation of receptor-tagged gene-targeting mouse lines, based on their enrichment in the functional imaging area (Olfr881: FDR $=0.007, \operatorname{LogFC}=2.55$ and Olfr1377: FDR $=$ $0.021, \operatorname{LogFC}=2.14)($ fig. $\mathrm{S} 12 \mathrm{~B}$ ) and their robust response to the odorant 4methylacetophenone in pS6-IP RNA-Seq experiments (Olfr1377: FDR $=3.43 e^{-26}$, LogFC $=$

3.18, Olfr881: FDR $\left.=7.37 e^{-23}, \operatorname{LogFC}=3.18\right)(\text { fig. S12C })^{65}$. Using Easi-CRISPR ${ }^{66}$, we inserted IRES-mKate 2 cassettes following the CDS of each OR to create Olfr1377-IRESmKate2 and Olfr881-IRES-mKate2 mice, in which OSNs expressing either Olfr1377 or Olfr881 also express the cytosolic fluorescent marker mKate2, labeling cell bodies in the OE 287 and glomeruli in the OB (fig. S12D). Whole-mount confocal imaging of the OB in genetargeted mice revealed mKate2-labeled glomeruli within the dorsal-central OB surface (Fig. 6A and fig. S13, A and B).

Examination of additional whole-mount epifluorescence images allowed us to further assess position and variance of both Olfr1377 and Olfr881 (fig. S14, Fig. 5C). Consistent with the singular expression of most ORs as two mirror-symmetric glomeruli, both gene-targeted mouse lines labeled two glomeruli per OB. The lateral Olfr1377 glomerulus $(n=10)$ was positioned centrally along on the AP axis and central-laterally within the ML axis imaging area, while the medial glomerulus $(n=6)$ was more posterior, ventral, and medial. The lateral Olfr881 glomerulus ( $n=9$ ) was positioned centrally along the ML axis and relatively posterior within the imaging area, while the medial glomerulus $(n=8)$ displayed a more variable position across the medioposterior quarter of the dorsal surface. The lateral Olfr1377 
300 glomerulus while the medial Olfr1377 glomerulus was distributed in an area nearly half the

301 size $(52.8 \%)$ of its Olfr881 counterpart.

302

303

Functional imaging of dorsal ORs

304 Expression of long-wavelength mKate2 as an OR-specific marker allowed for functional 305 characterization of Olfr1377 and Olfr881 glomeruli by crossing the generated mouse lines to 306 OSN-specific driver lines expressing a GCaMP $\mathrm{Ca}^{2+}$ reporter. For maximal imaging 307 sensitivity, we crossed each mKate2 line to the OMP-IRES-tTA driver line ${ }^{67}$ and the tetO308 GCaMP6s reporter line ${ }^{68}$. In the resulting triple crosses, we readily located the lateral mKate2309 tagged glomeruli on the dorsal functional imaging surface (Fig. 6A) and imaged odorant310 evoked GCaMP6s signals from these and neighboring glomeruli using dual-wavelength two311 photon imaging in anesthetized mice. Consistent with our pS6-IP in vivo data (fig. S12C), both 312 Olfr1377 and Olfr881 exhibited robust responses to low concentrations of 4-

313 methylacetophenone, with Olfr1377 exhibiting a stronger response than Olfr881 (Fig. 6, B and 314 C). In addition to 4-methylacetophenone, we also tested a large odorant panel including 315 multiple cyclic ketones structurally related to 4-methylacetophenone, as well as more diverse 316 odorants, all at relatively low concentrations. From this panel, we identified multiple new, high317 affinity ligands for each OR, including many cyclic ketones, and ultimately uncovered 318 overlapping but distinct response spectra for Olfr1377 and Olfr881 (Fig. 6C, fig. S15C). For example, Olfr1377 showed strong responses to p-anisaldehyde, acetophenone, and the aliphatic ketone 4-methyl-3-penten-2-one, while Olfr881 proved unresponsive to these 321 odorants.

322 Interestingly, Olfr1377 (but not Olfr881) exhibited an exceptionally strong response to 4methoxyacetophenone, with a brief (2 s) presentation of $\sim 0.4 \mathrm{nM} 4$-methoxyacetophenone 324 eliciting long-term activation and desensitization (fig. S15, A and B). Additional concentration 
screening suggested an in vivo response threshold of $\sim 10^{-13} \mathrm{M}$ 4-methoxyacetophenone for

326 the Olfr1377 glomerulus (Fig. 6D and fig. S15D). To complement our in vivo imaging and

327 pS6-IP RNA-Seq analyses and further evaluate the sensitivity of Olfr1377 to 4-

328 methylacetophenone and 4-methoxyacetphenone, we additionally expressed Olfr1377 in

329 Hana3a cells and examined luciferase responses to different concentrations of 4-

330 methoxyacetophenone, 4-methylacetophenone, 2'-hydroxyacetophenone, and

331 acetophenone ${ }^{69,70}$. Reinforcing our in vivo results, Olfr1377 responded to all four odorants

332 (Fig. 6E). Moreover, Olfr1377 responded to 4-methoxyacetophenone at just $1 \mathrm{nM}$ and saturated at $100 \mathrm{nM}$ in vitro, a response orders-of-magnitude more sensitive than the response to any other odorant tested. Collectively, these findings identify 4methoxyacetophenone-Olfr1377 as a ligand-receptor interaction with exceptionally high 336 affinity.

\section{DISCUSSION}

A central goal of neurobiology is to understand how sensory systems interpret the external world ${ }^{71}$. The visual and auditory sensory maps feature topographic maps which organize and process stimuli such as light position and sound frequency. In vertebrates, the organization of the olfactory topographic map remains largely unknown, and understanding how OR glomeruli are organized can reveal how inputs are transformed into output signals for the olfactory cortex as well as which aspects of olfactory input are important ${ }^{72,73}$. In Drosophila, glomeruli have been individually linked to specific ORs, allowing for a comprehensive understanding of how sensory information is organized within the first olfactory relay ${ }^{73-75}$. This key map has served as a foundation for critical studies regarding sensory processing, olfactory receptor neuron-targeting factors, the propagation of information to higher-order neurons, and the cellular composition of the antennal lobe ${ }^{76-79}$. 
Here, we demonstrate a unique application of target capture sequencing, enriching low-

abundance target transcripts found in the axon terminals of OSNs and comparing postenrichment abundances across samples. Our high-throughput approach enabled us to generate the first repertoire-scale dataset for OR glomeruli positions. This approach also allowed us to create a three-dimensional positional estimate for the glomeruli of a majority of the OR repertoire with precision within the ranges previously observed for positional variability between animals ${ }^{5-8,23}$.

With a systematic, anatomical mapping of OR identities to OB glomeruli, we can begin to understand which features of sensory information impact organizational strategies utilized within the mouse $\mathrm{OB}$, the central circuit of olfaction. We identified functional odor-OR relationships at the peripheral interface using the in vivo pS6-IP RNA-Seq deorphanization approach. By identifying the set of ORs within functionally imaged glomeruli future studies will be able to determine how $\mathrm{OB}$ circuits transform peripheral input into neural representations of odors at the OR level. Additional gene targeted mouse lines amenable to functional imaging will also enable us to better understand interglomerular connectivity and how patterns of inhibition are established between nearby glomeruli. The combination of our newly generated OR-OB positional data with ligand-receptor deorphanization data will therefore enable largescale investigations into how the odor space relates to topographical features in the OB and will facilitate the development of unified, systematic models for how odorants are processed, interpreted, and transformed into behavior.

\section{ACKNOWLEDGMENTS:}

We thank members of the Matsunami lab for thoughtful discussions and feedback. Luis R. Saraiva (SIDRA Institute), Antonio Scialdone (Helmholtz Zentrum München), and Mayra L. Ruiz Tejada Segura (Helmholtz Zentrum München) provided helpful advice on mapping OR 
expression and spatial transcriptomics. We thank Izumi Fukunaga (Okinawa Institute of

376 Science and Technology) for providing constructive comments on the preprint which helped

377 improve the clarity of the manuscript. We thank Sayan Mukherjee (Duke University) for

378 guidance on the combination of single dimension transcriptomic data into a three-dimensional

379 model. We thank C. Ron Yu (Stowers Institute, Univ. of Kansas) for providing OMP-IRES-tTA

380 mice. We thank Shelby Priest (Duke University) for assistance with critical reading and

381 feedback. Hiroaki Matsunami is supported by National Science Foundation grants 2014217

382 and 1556207 as well as National Institute on Deafness and Other Communication Disorders

383 grants R01DC014423 and R01DC016224. Matt Wachowiak is supported by National Science

384 Foundation grants 2014217 and 1555919 as well as National Institute of Neurological

385 Disorders and Stroke grant R01NS109979. Kevin Zhu is supported by National Institute on

386 Deafness and Other Communication Disorders grant F31DC017394. Shawn Burton is

387 supported by National Institute of Mental Health grant F32MH115448. Claire de March is

388 supported by National Institute on Deafness and Other Communication Disorders grant 389 K99DC018333.

390

391 AUTHOR CONTRIBUTIONS:

392 Conceptualization: KWZ, MW, HM

393 Methodology: KWZ, SDB, JDS, MW, HM

394 Investigation: KWZ, SDB, MHN, MW, HM

395 Visualization: KWZ, SDB, MHN, CAdM, MW, HM

396 Funding acquisition: KWZ, SDB, MW, HM

397 Project administration: MW, HM

398 Supervision: MW, HM

399 Writing - original draft: KWZ, SDB, MW, HM 
400 Writing - review \& editing: KWZ, SDB, MHN, JDS, CAdM, MW, HM

401

402 COMPETING INTERESTS:

$403 \mathrm{HM}$ has received royalties from Chemcom. HM has received research grants from Givaudan.

404 HM has received consultant fees from Kao. The remaining authors declare no competing 405 interests. 
REFERENCES:

408 1. Buck, L. B. Information coding in the vertebrate olfactory system. Annu. Rev. Neurosci. $409 \quad 19,517-544(1996)$.

410 2. Takeuchi, H. \& Sakano, H. Neural map formation in the mouse olfactory system. Cell. $411 \quad$ Mol. Life Sci. 71, 3049-3057 (2014).

412 3. Potter, S. M. et al. Structure and emergence of specific olfactory glomeruli in the 413 mouse. J. Neurosci. 21, 9713-9723 (2001).

414 4. Pinching, A. J. \& Powell, T. P. The neuropil of the glomeruli of the olfactory bulb. J. Cell 415 Sci. 9, 347-377 (1971).

416 5. Ressler, K. J., Sullivan, S. L. \& Buck, L. B. Information coding in the olfactory system: Evidence for a stereotyped and highly organized epitope map in the olfactory bulb. Cell 79, 1245-1255 (1994).

6. Vassar, R. et al. Topographic organization of sensory projections to the olfactory bulb. Cell 79, 981-991 (1994).

421 7. Mombaerts, P. et al. Visualizing an olfactory sensory map. Cell 87, 675-686 (1996).

422 8. Zapiec, B. \& Mombaerts, P. Multiplex assessment of the positions of odorant receptor423 specific glomeruli in the mouse olfactory bulb by serial two-photon tomography. Proc. Natl. Acad. Sci. 112, E5873-82 (2015).

9. Rubin, B. D. \& Katz, L. C. Optical imaging of odorant representations in the mammalian olfactory bulb. Neuron 23, 499-511 (1999).

10. Friedrich, R. W. \& Korsching, S. I. Combinatorial and Chemotopic Odorant Coding in the Zebrafish Olfactory Bulb Visualized by Optical Imaging. Neuron 18, 737-752 (1997).

11. Wachowiak, M. \& Cohen, L. B. Representation of odorants by receptor neuron input to the mouse olfactory bulb. Neuron 32, 723-735 (2001). 
432 12. Bozza, T., McGann, J. P., Mombaerts, P. \& Wachowiak, M. In vivo imaging of neuronal activity by targeted expression of a genetically encoded probe in the mouse. Neuron $\mathbf{4 2}$, 9-21 (2004).

13. Oka, Y. et al. Odorant Receptor Map in the Mouse Olfactory Bulb: In Vivo Sensitivity and Specificity of Receptor-Defined Glomeruli. Neuron 52, 857-869 (2006).

14. Zheng, C., Feinstein, P., Bozza, T., Rodriguez, I. \& Mombaerts, P. Peripheral Olfactory Projections Are Differentially Affected in Mice Deficient in a Cyclic Nucleotide-Gated Channel Subunit. Neuron 26, 81-91 (2000).

15. Smear, M., Resulaj, A., Zhang, J., Bozza, T. \& Rinberg, D. Multiple perceptible signals from a single olfactory glomerulus. Nat. Neurosci. 16, 1687-1691 (2013).

16. Strotmann, J. et al. Local permutations in the glomerular array of the mouse olfactory bulb. J. Neurosci. 20, 6927-6938 (2000).

17. Hulst, C. D. et al. MouSensor : A Versatile Genetic Platform to Create Super Sniffer Mice for Studying Human Odor Coding Article MouSensor : A Versatile Genetic Platform to Create Super Sniffer Mice for Studying Human Odor Coding. Cell Rep. 16, $1-11(2016)$.

18. Bozza, T., Feinstein, P., Zheng, C. \& Mombaerts, P. Odorant receptor expression defines functional units in the mouse olfactory system. J. Neurosci. 22, 3033-3043 (2002).

19. Grosmaitre, X. et al. SR1, a mouse odorant receptor with an unusually broad response profile. J. Neurosci. 29, 14545-52 (2009).

20. Shirasu, M. et al. Olfactory receptor and neural pathway responsible for highly selective sensing of musk odors. Neuron 81, 165-178 (2014).

21. Saito, H. et al. Immobility responses are induced by photoactivation of single glomerular species responsive to fox odour TMT. Nat. Commun. 8, 1-10 (2017). 
457 22. Mizrahi, A., Matsunami, H. \& Katz, L. C. An imaging-based approach to identify ligands 458 for olfactory receptors. Neuropharmacology 47, 661-668 (2004).

459 23. Miyamichi, K., Serizawa, S., Kimura, H. \& Sakano, H. Continuous and Overlapping Expression Domains of Odorant Receptor Genes in the Olfactory Epithelium Determine 461 462

24. Feinstein, P., Bozza, T., Rodriguez, I., Vassalli, A. \& Mombaerts, P. Axon guidance of mouse olfactory sensory neurons by odorant receptors and the B2AR. Cell 117, 833846 (2004).

25. Li, J., Ishii, T., Feinstein, P. \& Mombaerts, P. Odorant receptor gene choice is reset by nuclear transfer from mouse olfactory sensory neurons. Nature 428, 393-399 (2004).

26. Tsuboi, A., Miyazaki, T., Imai, T. \& Sakano, H. Olfactory sensory neurons expressing class I odorant receptors converge their axons on an antero-dorsal domain of the olfactory bulb in the mouse. Eur. J. Neurosci. 23, 1436-1444 (2006).

27. Tsuboi, A. et al. Olfactory neurons expressing closely linked and homologous odorant receptor genes tend to project their axons to neighboring glomeruli on the olfactory bulb. J. Neurosci. 19, 8409-8418 (1999).

28. Serizawa, S. et al. Mutually exclusive expression of odorant receptor transgenes. Nat. Neurosci. 3, 687-693 (2000).

29. Bozza, T. et al. Mapping of Class I and Class II Odorant Receptors to Glomerular Domains by Two Distinct Types of Olfactory Sensory Neurons in the Mouse. Neuron 61, 220-233 (2009).

30. Lin, D. M. et al. Spatial patterns of gene expression in the olfactory bulb. Proc. Natl. Acad. Sci. U. S. A. 101, 12718-12723 (2004).

31. Mortazavi, A., Williams, B. A., McCue, K., Schaeffer, L. \& Wold, B. Mapping and 
quantifying mammalian transcriptomes by RNA-Seq. Nat. Methods 5, 621-628 (2008).

32. Zou, D.-J. et al. Postnatal Refinement of Peripheral Olfactory Projections. Science (80-. ). 304, 1976-1979 (2004).

33. Stahl, P. et al. Visualization and analysis of gene expression in tissue sections by spatial transcriptomics. Science (80-. ). 353, (2016).

34. Mercer, T. R. et al. Targeted sequencing for gene discovery and quantification using RNA CaptureSeq. Nat. Protoc. 9, 989-1009 (2014).

35. Ressler, K. J., Sullivan, S. L. \& Buck, L. B. A zonal organization of odorant receptor gene expression in the olfactory epithelium. Cell 73, 597-609 (1993).

36. Vassar, R., Ngai, J. \& Axel, R. Spatial segregation of odorant receptor expression in the mammalian olfactory epithelium. Cell 74, 309-318 (1993).

37. Astic, L. \& Saucier, D. Anatomical mapping of the neuroepithelial projection to the olfactory bulb in the rat. Brain Res. Bull. 16, 445-454 (1986).

38. Tan, L. \& Xie, X. S. A near-complete spatial map of olfactory receptors in the mouse main olfactory epithelium. Chem. Senses 43, 427-432 (2018).

39. Zapiec, B. \& Mombaerts, P. The Zonal Organization of Odorant Receptor Gene Choice in the Main Olfactory Epithelium of the Mouse. Cell Rep. 30, 4220-4234 (2020).

40. Becht, E. et al. Dimensionality reduction for visualizing single-cell data using UMAP. Nat. Biotechnol. 37, 38-47 (2019).

41. Schaefer, M. L., Finger, T. E. \& Restrepo, D. Variability of position of the P2 glomerulus within a map of the mouse olfactory bulb. J. Comp. Neurol. 436, 351-362 (2001).

42. Mayer, C. D., Lorent, J. \& Horgan, G. W. Exploratory analysis of multiple omics datasets using the adjusted RV coefficient. Stat. Appl. Genet. Mol. Biol. 10, (2011).

43. Mori, K. \& Sakano, H. How is the olfactory map formed and interpreted in the mammalian brain? Annu. Rev. Neurosci. 34, 467-499 (2011). 
507 44. Murthy, V. N. Olfactory maps in the brain. Annu. Rev. Neurosci. 34, 233-258 (2011).

508 45. Zapiec, B., Christian Bressel, O., Khan, M., Walz, A. \& Mombaerts, P. Neuropilin-1 and 509 the Positions of Glomeruli in the Mouse Olfactory Bulb. eNeuro 3, 1-19 (2016).

510 46. Soucy, E., Albeanu, D., Antoniu, F., Murthy, V. \& Meister, M. Precision and diversity in 511 an odor map on the olfactory bulb. Nat. Neurosci. 12, 1351-1360 (2009).

512 47. Pacifico, R., Dewan, A., Cawley, D., Guo, C. \& Bozza, T. An olfactory subsystem that 513

48. Nakashima, A. et al. Agonist-independent GPCR activity regulates anterior-posterior mediates high-sensitivity detection of volatile amines. Cell Rep. 2, 76-88 (2012). targeting of olfactory sensory neurons. Cell 154, 1314-1325 (2013).

49. Wang, F., Nemes, A., Mendelsohn, M. \& Axel, R. Odorant Receptors Govern the Formation of a Precise Topographic Map. Cell 93, 47-60 (1998).

50. Feinstein, P. \& Mombaerts, P. A contextual model for axonal sorting into glomeruli in the mouse olfactory system. Cell 117, 817-831 (2004).

51. Glusman, G. et al. The olfactory receptor gene superfamily: Data mining, classification, and nomenclature. Mamm. Genome 11, 1016-1023 (2000).

52. Olender, T., Jones, T. E. M., Bruford, E. \& Lancet, D. A unified nomenclature for vertebrate olfactory receptors. BMC Evol. Biol. 20, 1-12 (2020).

53. Grantham, R. Amino Acid Difference Formula to Help Explain Protein Evolution. Science (80-. ). 185, 862-864 (1974).

54. Rasmussen, S. G. F. et al. Crystal structure of the $\beta 2$ adrenergic receptor-Gs protein complex. Nature 477, 549-557 (2011).

55. Kato, A., Katada, S. \& Touhara, K. Amino acids involved in conformational dynamics and G protein coupling of an odorant receptor: Targeting gain-of-function mutation. J. Neurochem. 107, 1261-1270 (2008).

56. Kato, A. \& Touhara, K. Mammalian olfactory receptors: Pharmacology, G protein 
coupling and desensitization. Cell. Mol. Life Sci. 66, 3743-3753 (2009).

\section{3}

57. De March, C. A., Kim, S. K., Antonczak, S., Goddard, W. A. \& Golebiowski, J. G protein-coupled odorant receptors: From sequence to structure. Protein Sci. 24, 15431548 (2015).

58. Imai, T., Suzuki, M. \& Sakano, H. Odorant Receptor-Derived cAMP Signals Direct Axonal Targeting. Science (80-. ). 313, 667-669 (2006).

59. Imai, T. et al. Pre-Target Axon Sorting Establishes the Neural Map Topography. Science (80-. ). 17, 585-590 (2009).

60. Assens, A. et al. Alteration of Nrp1 signaling at different stages of olfactory neuron maturation promotes glomerular shifts along distinct axes in the olfactory bulb. Development 143, 3817-3825 (2016).

61. Johnson, G. A. et al. Waxholm Space: An image-based reference for coordinating mouse brain research. Neuroimage 53, 365-372 (2010).

62. Egozcue, J. J., Pawlowsky-Glahn, V., Mateu-Figueras, G. \& Barceló-Vidal, C. Isometric Logratio Transformations for Compositional Data Analysis. Math. Geol. 35, 279-300 (2003).

63. Silverman, J. D., Washburne, A. D., Mukherjee, S. \& David, L. A. A phylogenetic transform enhances analysis of compositional microbiota data. Elife 6, 1-20 (2017).

64. Ma, M. \& Shepherd, G. M. Functional mosaic organization of mouse olfactory receptor neurons. Proc. Natl. Acad. Sci. U. S. A. 97, 12869-12874 (2000).

65. Jiang, Y. et al. Molecular profiling of activated olfactory neurons identifies odorant receptors responding to odors in vivo. Nat. Neurosci. 18, 1446-54 (2015).

66. Quadros, R. M. et al. Easi-CRISPR: A robust method for one-step generation of mice carrying conditional and insertion alleles using long ssDNA donors and CRISPR ribonucleoproteins. Genome Biol. 18, 1-15 (2017). 
557 67. Yu, C. R. et al. Spontaneous neural activity is required for the establishment and 558 maintenance of the olfactory sensory map. Neuron 42, 553-566 (2004).

559 68. Wekselblatt, J. B., Flister, E. D., Piscopo, D. M. \& Niell, C. M. Large-scale imaging of $560 \quad$ cortical dynamics during sensory perception and behavior. J. Neurophysiol. 115, 2852$5612866(2016)$.

562 69. Saito, H., Kubota, M., Roberts, R. W., Chi, Q. \& Matsunami, H. RTP family members 563 induce functional expression of mammalian odorant receptors. Cell 119, 679-691 564 (2004).

565 70. Zhuang, H. \& Matsunami, H. Evaluating cell-surface expression and measuring 566 activation of mammalian odorant receptors in heterologous cells. Nat. Protoc. 3, 1402$567 \quad 1413(2008)$.

568 71. Ernst, M. O. \& Bülthoff, H. H. Merging the senses into a robust percept. Trends Cogn. 569 Sci. 8, 162-169 (2004).

$57072 . \quad$ Vosshall, L. B. \& Stocker, R. F. Molecular architecture of smell and taste in Drosophila. $571 \quad$ Annu. Rev. Neurosci. 30, 505-533 (2007).

572 73. Grabe, V., Strutz, A., Baschwitz, A., Hansson, B. S. \& Sachse, S. Digital in vivo 3D 573 atlas of the antennal lobe of Drosophila melanogaster. J. Comp. Neurol. 523, 530-544 574 (2015).

575 74. Laissue, P. P. et al. Three-Dimensional Reconstruction of the Antennal Lobe in. J. 576 Comp. Neurol. 552, 543-552 (1999).

577 75. Hallem, E. A. \& Carlson, J. R. Coding of Odors by a Receptor Repertoire. Cell 125, $578 \quad 143-160(2006)$.

579 76. Chou, Y. H. et al. Diversity and wiring variability of olfactory local interneurons in the $580 \quad$ Drosophila antennal lobe. Nat. Neurosci. 13, 439-449 (2010).

581 77. Olsen, S. R., Bhandawat, V. \& Wilson, R. I. Divisive normalization in olfactory 
population codes. Neuron 66, 287-299 (2010).

583 78. Jeanne, J. M., Fişek, M. \& Wilson, R. I. The Organization of Projections from Olfactory Glomeruli onto Higher-Order Neurons. Neuron 98, 1198-1213.e6 (2018).

79. Tanaka, N. K., Endo, K. \& Ito, K. Organization of antennal lobe-associated neurons in adult Drosophila melanogaster brain. J. Comp. Neurol. 520, 4067-4130 (2012).

80. Köster, J. \& Rahmann, S. Snakemake-a scalable bioinformatics workflow engine. Bioinformatics 28, 2520-2522 (2012).

81. Dobin, A. et al. STAR: Ultrafast universal RNA-seq aligner. Bioinformatics 29, 15-21 (2013).

82. Li, B. \& Dewey, C. N. RSEM: accurate transcript quantification from RNA-Seq data with or without a reference genome. BMC Bioinformatics 12, 323 (2011).

83. Olender, T., Nativ, N. \& Lancet, D. HORDE: Comprehensive Resource for Olfactory Receptor Genomics. in Olfactory Receptors: Methods and Protocols (ed. Crasto, C. J.) 23-38 (Humana Press, 2013).

84. de March, C. A. et al. Odorant Receptor 7D4 Activation Dynamics. Angew. Chemie Int. Ed. 57, 4554-4558 (2018).

85. McCarthy, D. J., Chen, Y. \& Smyth, G. K. Differential expression analysis of multifactor RNA-Seq experiments with respect to biological variation. Nucleic Acids Res. 40, 42884297 (2012).

86. Zhuang, H. \& Matsunami, H. Synergism of accessory factors in functional expression of mammalian odorant receptors. J. Biol. Chem. 282, 15284-15293 (2007).

87. Saito, H., Chi, Q., Zhuang, H., Matsunami, H. \& Mainland, J. D. Odor coding by a Mammalian receptor repertoire. Sci. Signal. 2, ra9-ra9 (2009).

88. Burton, S. D., Wipfel, M., Guo, M., Eiting, T. P. \& Wachowiak, M. A Novel Olfactometer for Efficient and Flexible Odorant Delivery. Chem. Senses 44, 173-188 (2019). 


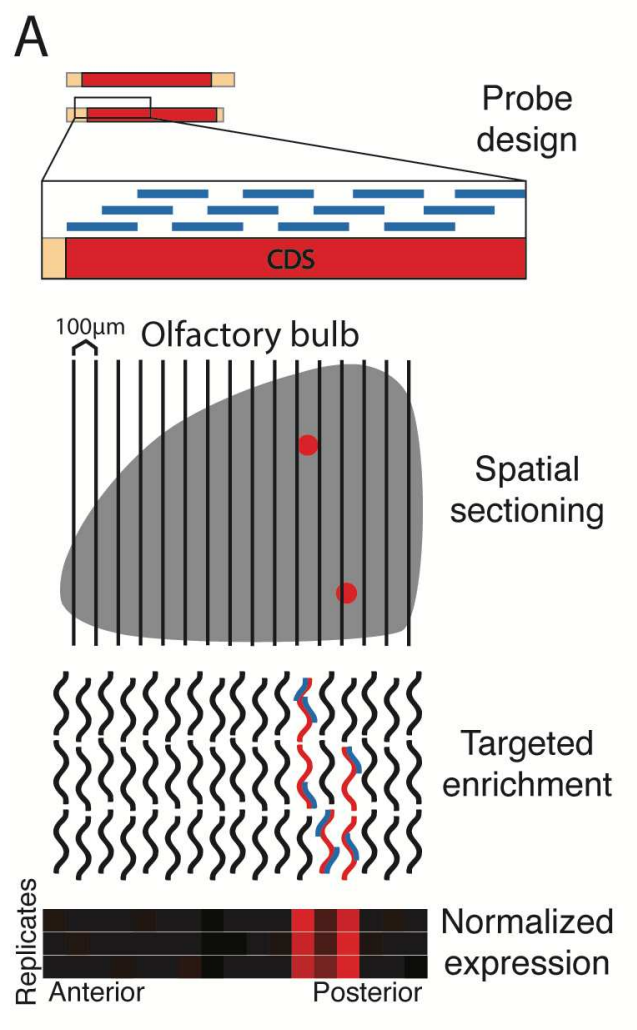

607

608

609

610

611

612

613

614

615

616

617

618

619
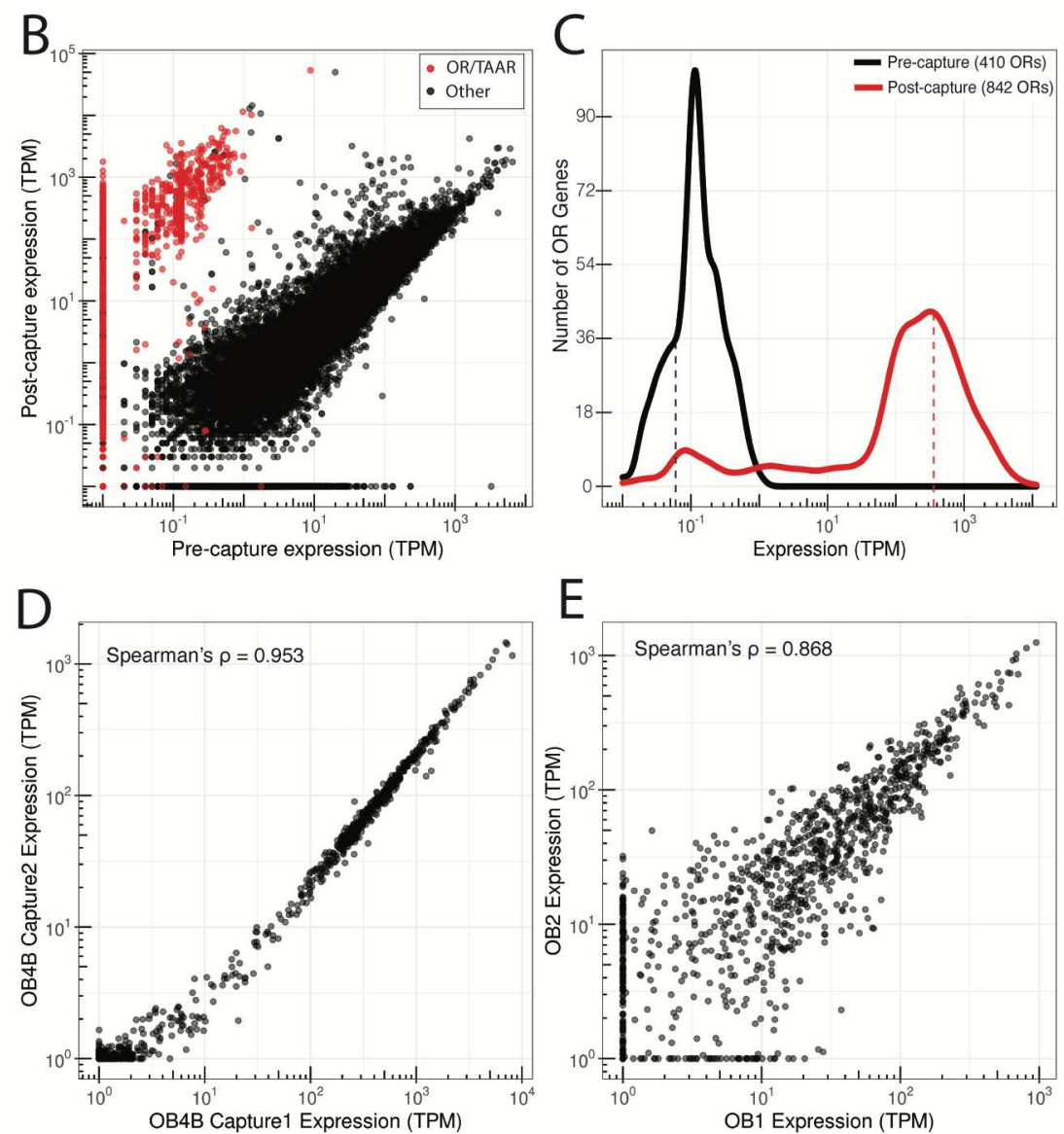

E

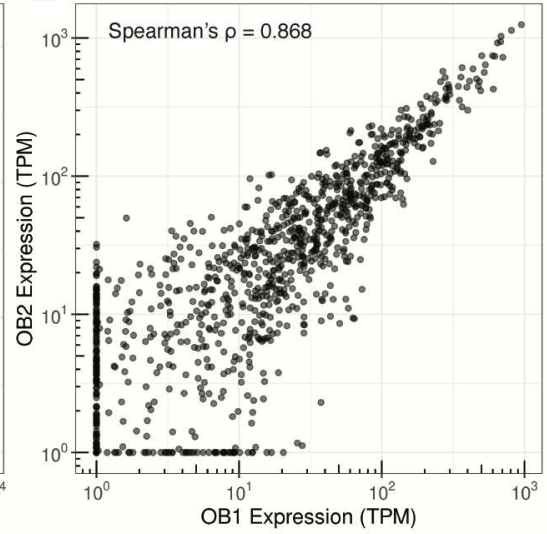

Fig. 1. Target capture sequencing consistently enriches $O R$ transcripts. (A)

Methodological overview for targeted enrichment of OR and TAAR transcripts from OB

sections. Briefly, RNA is extracted from OB tissue and used for cDNA synthesis and library

preparation. Capture probes are designed against coding sequences (CDS) of interest,

enabling enrichment of target genes following binding and washing steps. RNA-Seq of

enriched libraries allows for high-throughput positional analyses when combined with

systematic tissue sectioning. (B) Pre- and post-capture normalized abundances (transcripts

per million; TPM) of intact OR and TAAR genes (red) and other intact genes (black) from a

whole OB. (C) Frequency distribution of OR gene abundances pre- and post-capture from a

whole OB. (D) Technical replicates demonstrating OR and TAAR gene abundances from

independent capture enrichments of the same whole-OB RNA. (E) Biological replicates

demonstrating OR and TAAR gene abundances from capture enrichment of whole-OB RNA 
620 samples from different animals. 


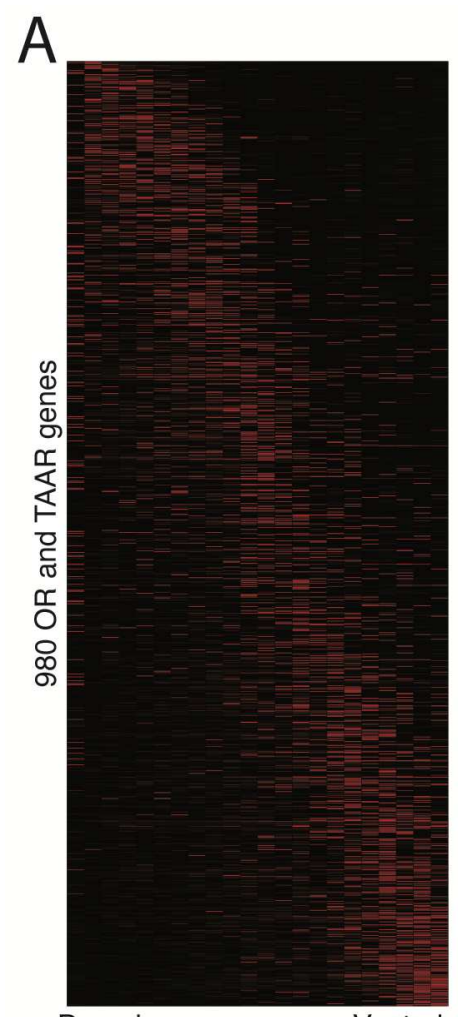

$C$
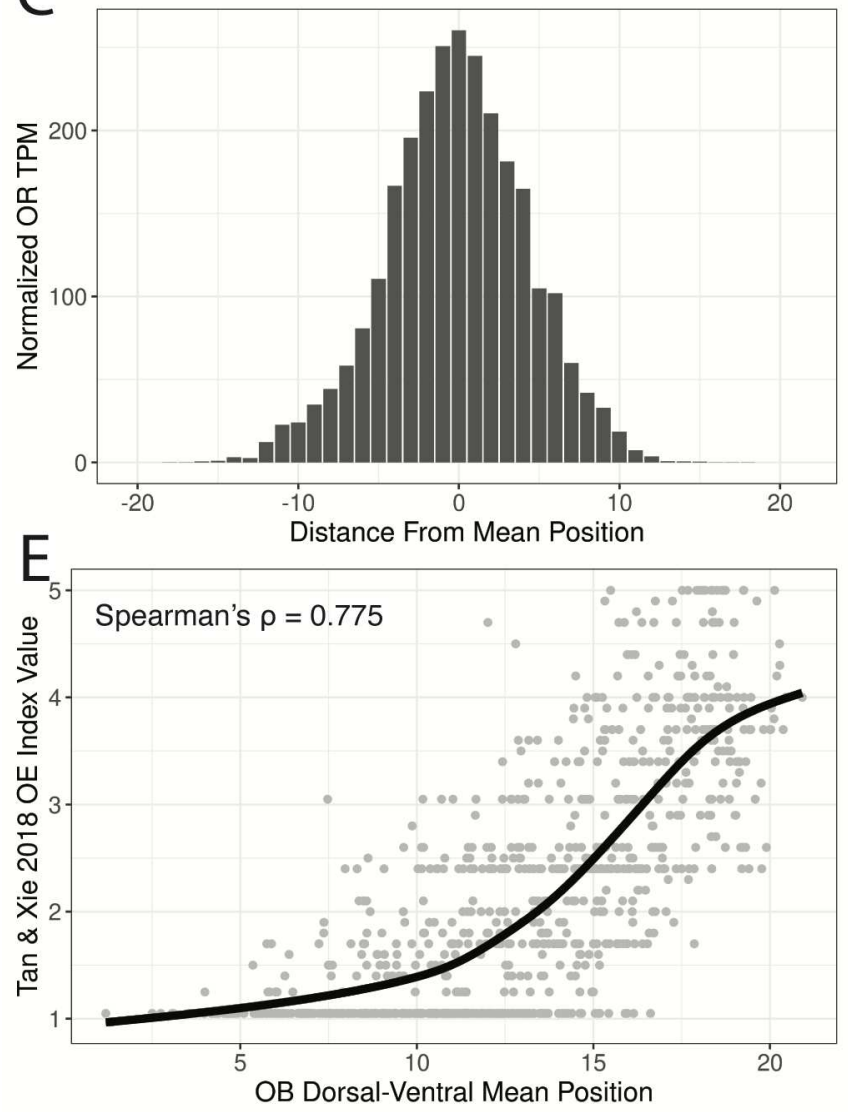

B

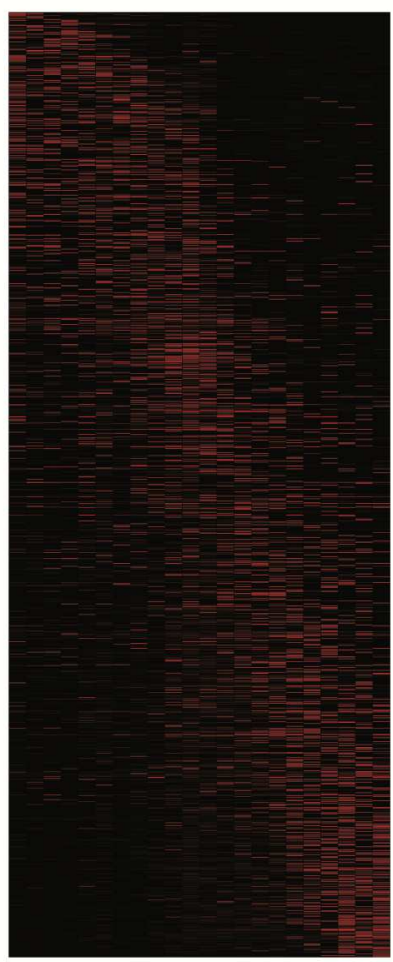

D

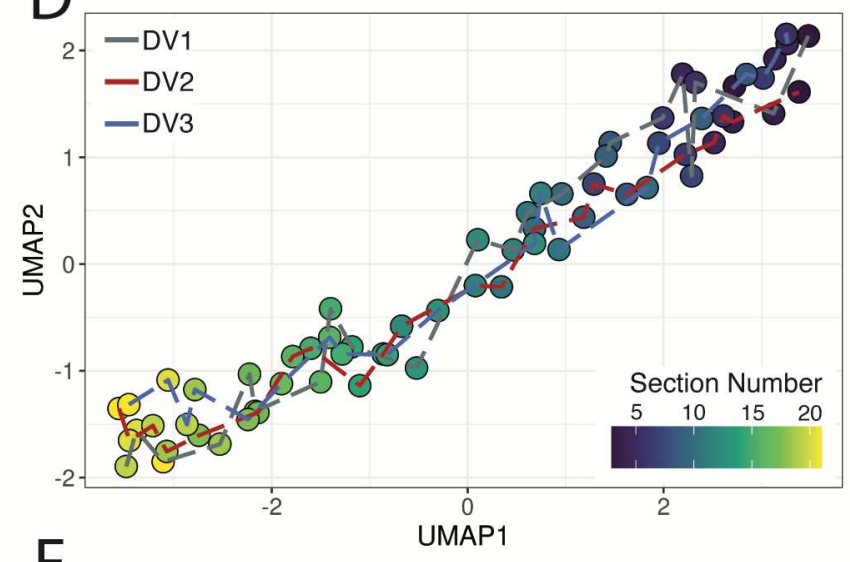

$\mathrm{F}$
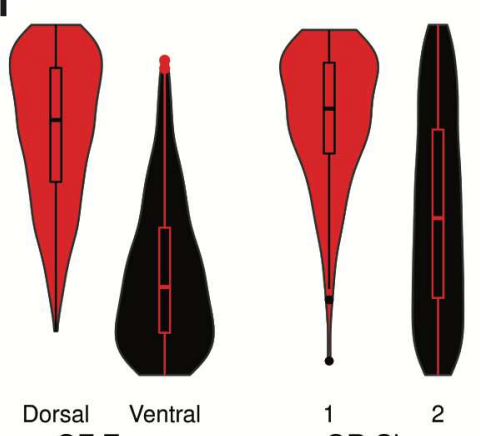

OE Zone

$\mathrm{p}=4.292 \mathrm{e}-95$

$n=441 \quad n=429$

Normalized TPM

1

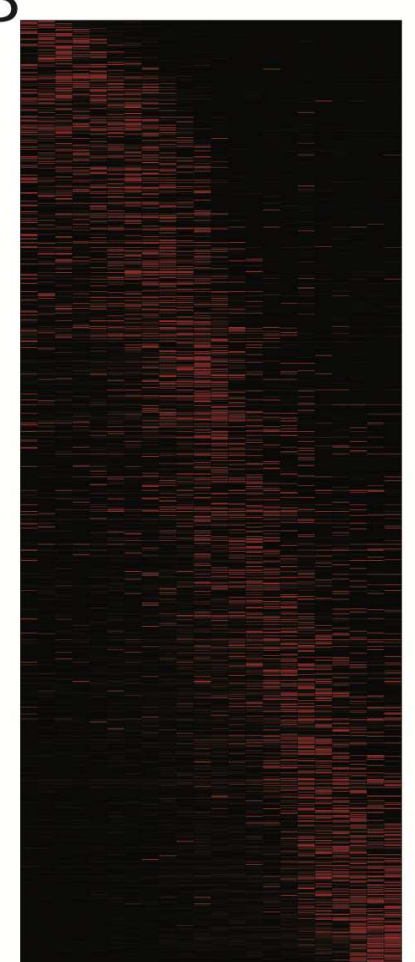

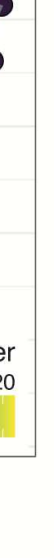


623 Heatmaps for 980 ORs and TAARs across 22 DV sections sorted by mean position of 624 expression from three replicate mice. (B) Merged representation of DV replicates. Ordering of 625 genes ( $Y$ axis) is consistent across all heatmaps. (C) Distribution of normalized TPM 626 (maximum observed value $=1$, minimum observed value $=0$ ) for all 980 ORs and TAARs 627 from position of mean expression. (D) UMAP projection of $22 \mathrm{DV}$ sections from all three 628 replicates. (E) Line is loess smoothed regression of OE DV index from Tan and Xie, Chem. 629 Senses 2018 across DV mean positions from our targeted spatial data. (F) Distribution of 630 ranked DV mean positions for the 980 ORs and TAARs by OE zone, OR class, and gene 631 family. Statistic is Mann-Whitney U-test. 


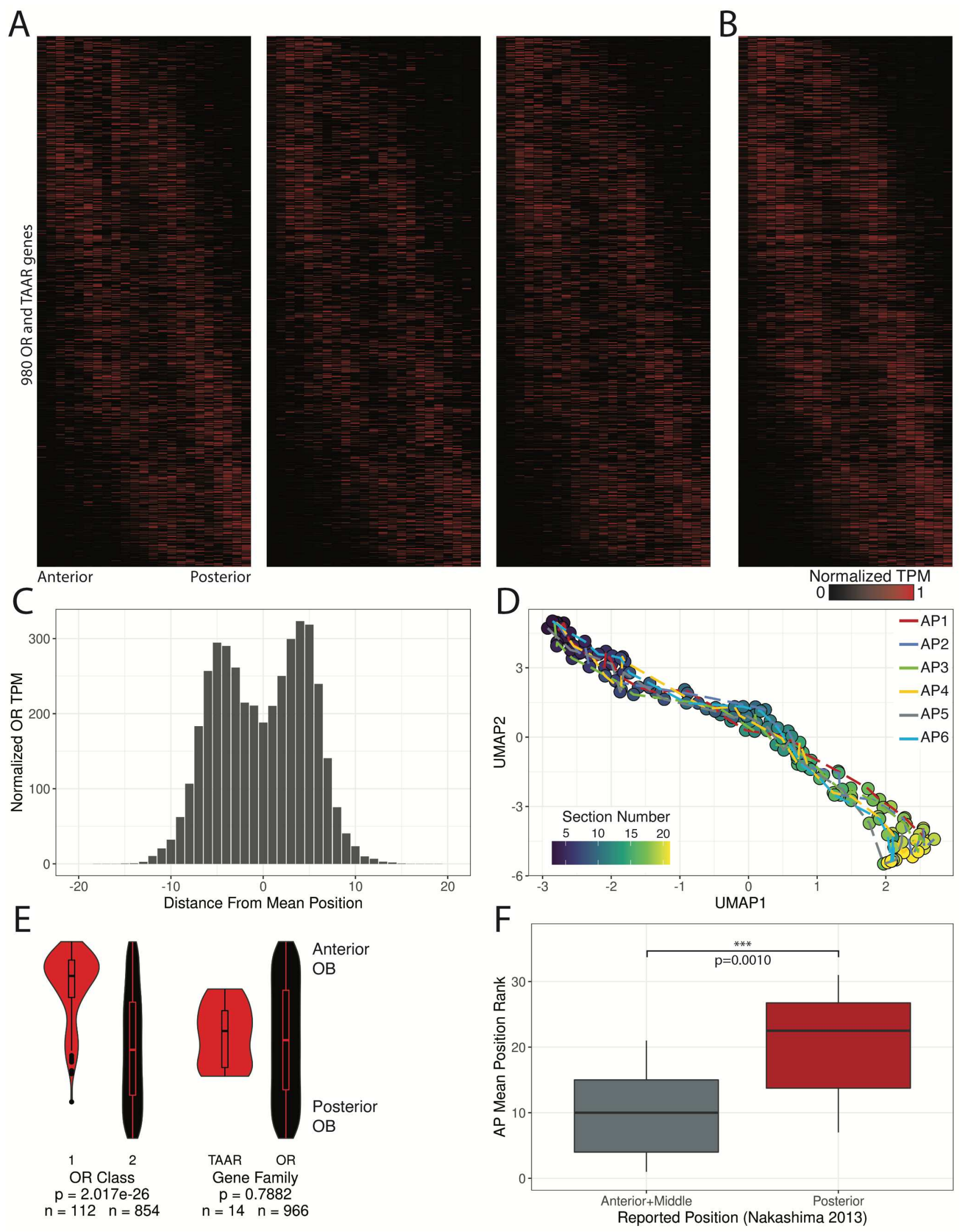

633 Fig. 3. Glomerular OR expression is bimodally distributed along the anteroposterior 
634 axis. (A) Heatmaps for 980 ORs and TAARs across 23 AP sections sorted by mean position 635 of expression from three replicate mice. (B) Merged representation from A. Ordering of genes 636 is consistent across all heatmaps. (C) Distribution of normalized TPM (maximum observed 637 value $=1$, minimum observed value $=0$ ) for all 980 ORs and TAARs from position of mean 638 expression. (D) UMAP projection of 23 AP sections across all six replicates. (E) Distribution of 639 ranked AP mean positions for the 980 ORs and TAARs by OR class and gene family. Statistic 640 is Mann-Whitney U-test. (F) Distribution of the ranked AP mean position for the set of ORs 641 cloned from the anterior and middle OB positions vs the posterior OB position from 642 Nakashima et al. Cell. 2013. Statistic is Mann-Whitney U-test. 


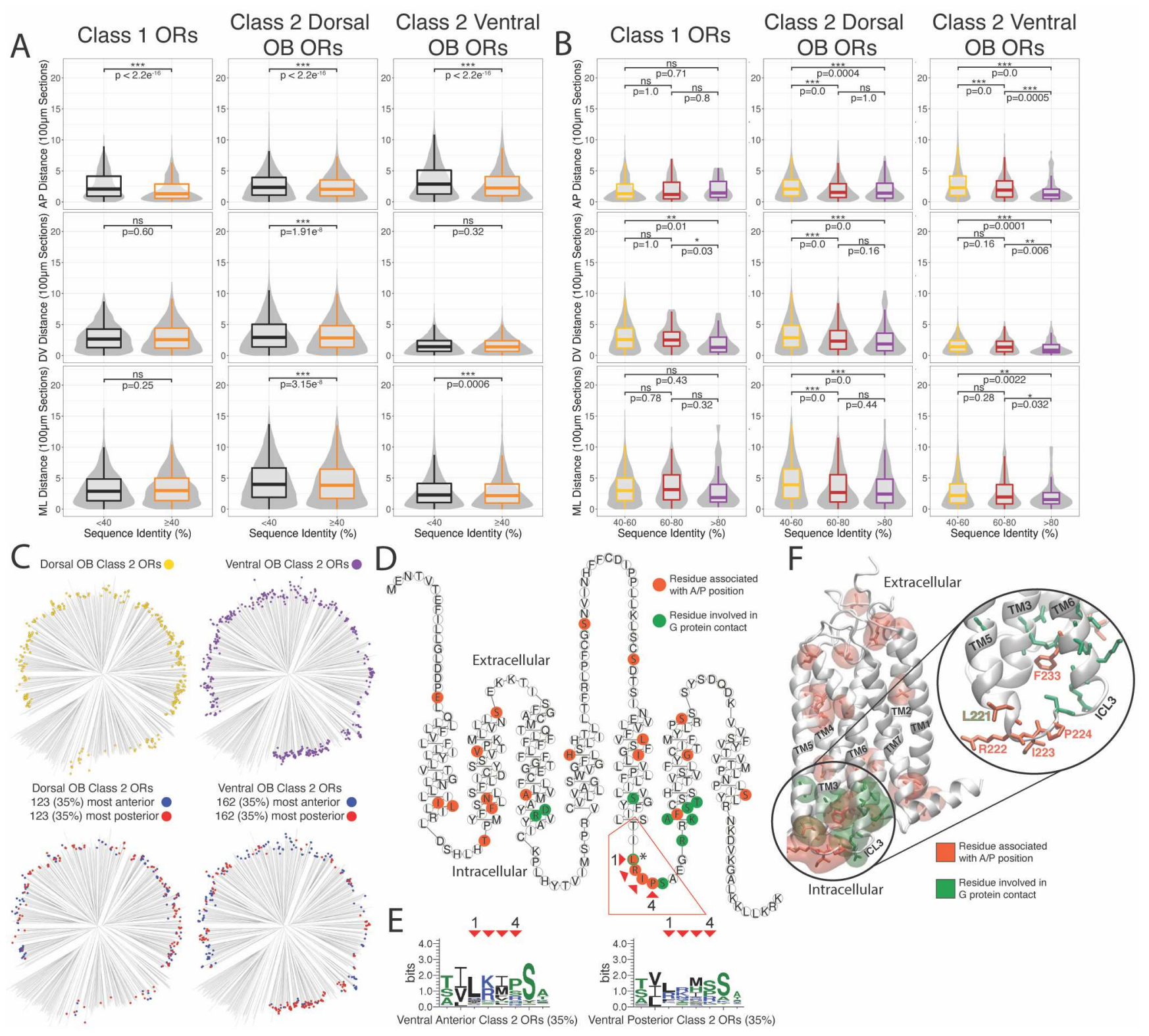

Fig. 4. OR sequence similarity correlates with glomerular position more strongly

646 among ventral than dorsal Class II ORs. (A) Pairwise comparisons between mean position

647 distance and OR protein sequence identity for ORs split by the family level homology cutoff

648 ( $\geq 40 \%$ in orange, $<40 \%$ in black) for the AP (top), DV (middle), and ML dimensions (bottom).

649 Statistic is Mann-Whitney U-test. (B) Pairwise comparisons between mean position distance

650 and OR protein sequence identity for ORs split by the family, subfamily, and orthologous OR

651 level homology cutoffs ( $40-60 \%$ in yellow, $60-80 \%$ in red, $\geq 80 \%$ in magenta) for the AP (top),

652 DV (middle), and ML dimensions (bottom). Statistic is Mann-Whitney U-test. (C) Phylogenetic 
653 tree of all Class II dorsal OB ORs (top left, $\mathrm{n}=354$ ), all Class II ventral OB ORs (top right, $\mathrm{n}=$ 654 464), the most anterior (35\%, blue) and most posterior (35\%, red) Class II ORs from the 655 dorsal (bottom left) and ventral OB (bottom right) DV zones. (D) Snakeplot of the Class II OR 656 consensus protein sequence; orange residues have significantly different physicochemical 657 properties for ventral, anterior or posterior, Class II ORs compared to all ventral Class II ORs. 658 Green residues indicate residues known to be involved in Class A GPCR activation through 659 contact with the $\mathrm{G}$ protein ( ${ }^{*}$ indicates the single residue which was identified as being both 660 associated with $\mathrm{G}$ protein contact and identified as significantly different for ventral, anterior, 661 Class II ORs). (E) Protein sequence logos for the four identified intracellular loop 3 residues 662 associated with ventral Class II anterior/posterior ORs depicting the conservation of specific 663 amino acid residues at each position. Red arrows indicate the specific residue within the 664 Class II OR consensus snakeplot (C) and the corresponding position in the sequence logo. 665 (F) Homology model of the mouse Class II consensus OR. Transmembrane helices (TM) are 666 numbered with residues associated with A/P positions (orange) and residues in contact with 667 the G protein (green) depicted in licorice with transparent regions indicating the residue 668 surface. Right, highlight of intracellular loop 3 (ICL3) where residues associated with A/P 669 positions are intermingled with the residues in contact with the $G$ protein. 

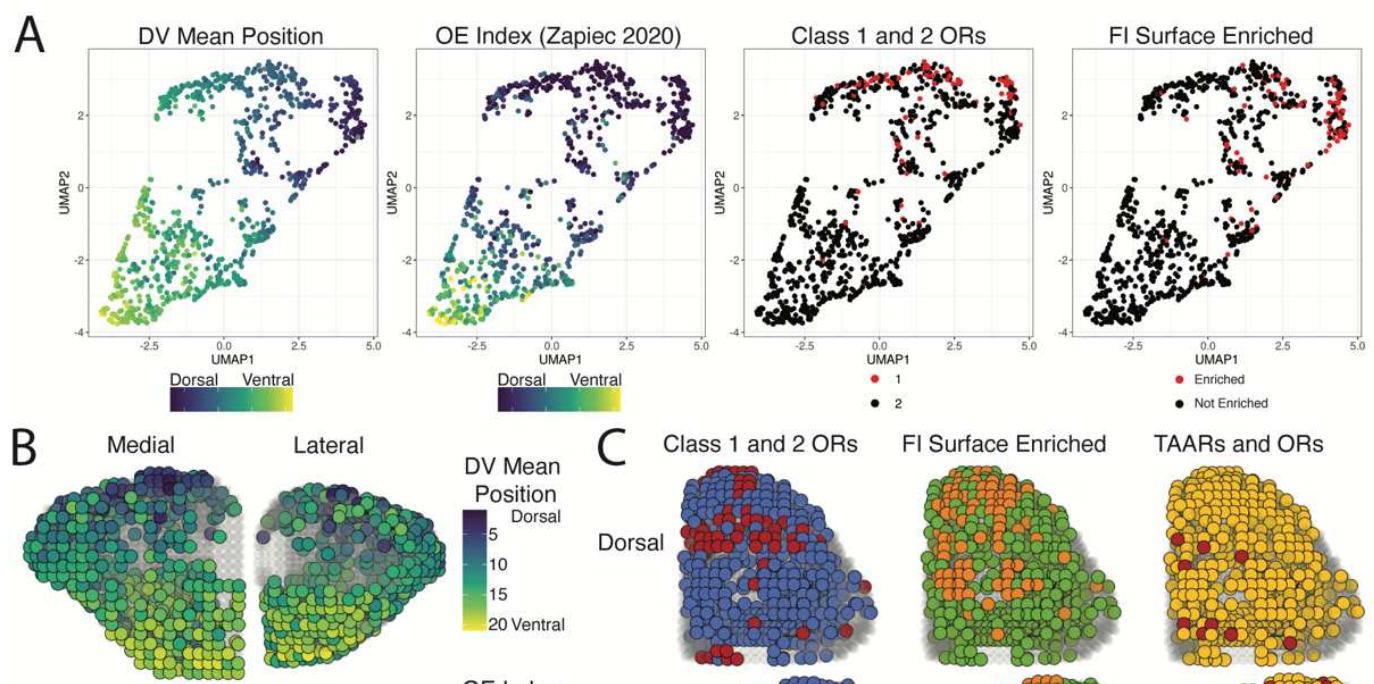

FI Surface Enriched
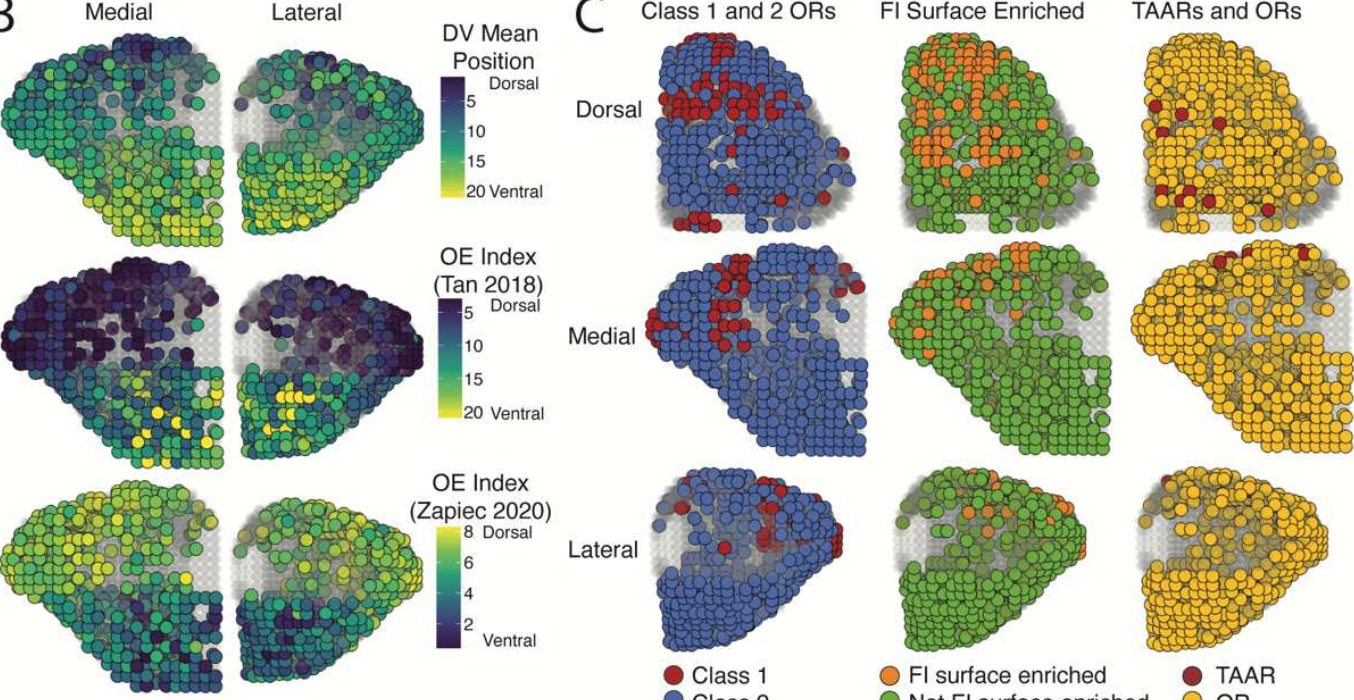

D Olfr1377
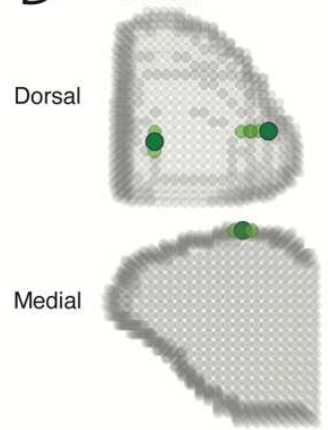

Lateral

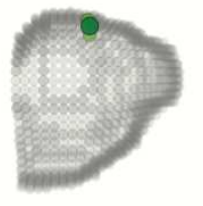

Best Probability

$\mathrm{E}$ High Probability

Olfr881
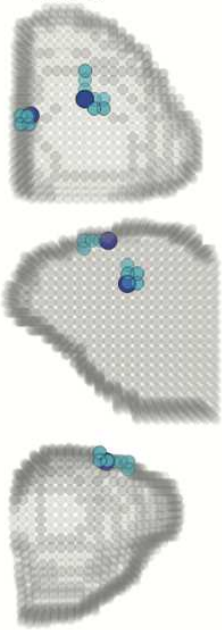

Best Probability High Probability

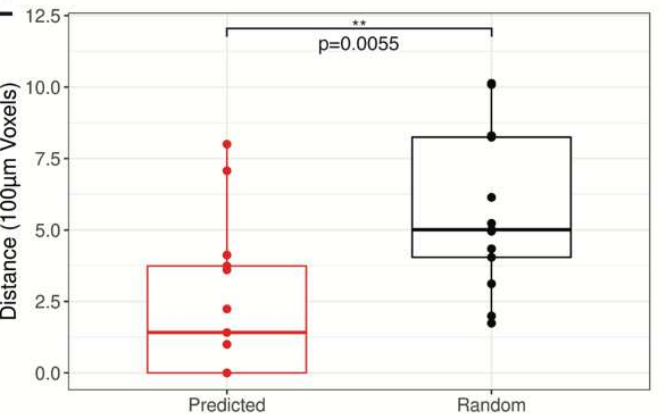

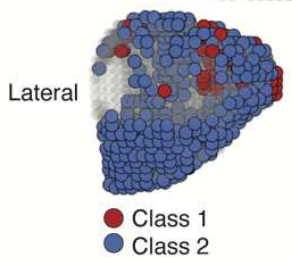

Olfr160/M72
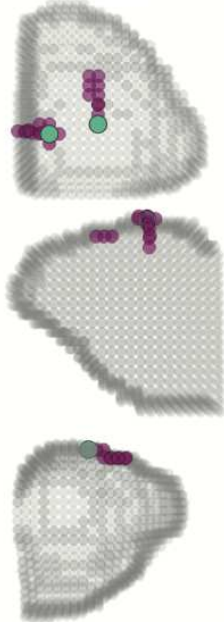

Best Probability High Probability
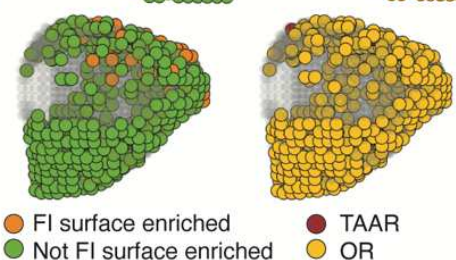

Not FI surface enriched OR

Olfr17/P2
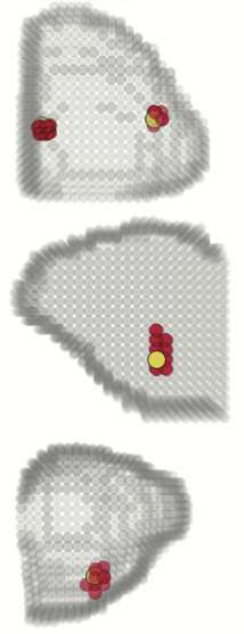

Best Probability High Probability
Olfr1507/MOR28
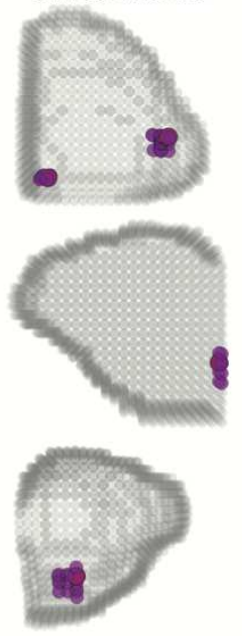

Best Probability - High Probability $\mathrm{F}$

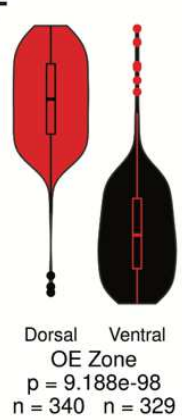

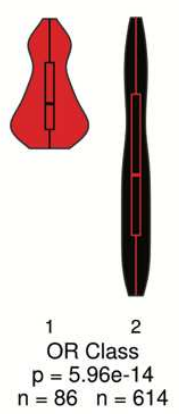

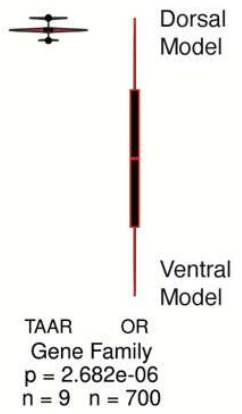


672 Fig. 5. A three-dimensional model for OR glomerular positions from combined single-

673 dimension targeted sequencing data. (A) UMAP projections of OR populations constructed 674 using the mean position from each individual replicate from the AP, DV, and ML dimensions.

675 DV mean position color reflects the calculated average mean position from all DV replicates. 676 OE Index (Zapiec 2020) refers to the OE index established by Zapiec and Mombaerts Cell 677 Reports 2020. FI Surface refers to the differential expression analysis calculated from dorsal 678 and ventral OB samples in this paper. (B) Three-dimensional predictions for the 709 ORs and 679 TAARs with DV mean positions (top) and with observed values in OE DV indices (middle and 680 bottom). (C) Three-dimensional predictions for the 709 ORs and TAARs with colors revealing 681 contrasting distributions of Class I vs Class II ORs, functional imaging surface enriched vs not 682 enriched ORs, and TAARs vs ORs. (D) Three-dimensional predictions for labeled ORs 683 generated in this study and from Zapiec and Mombaerts PNAS 2015. High probability 684 positions indicate the set of adjacent voxels containing highly ranked probabilities for that OR 685 with the best probability color indicates the voxel with the highest ranked probability within 686 that cluster of voxels. (E) Distance between predicted OR position and OR positions 687 determined from gene-targeted mouse lines compared to 50 random ORs from the same DV 688 zone (15 ORs used for unusual zone) and side of the OB. Statistic is Mann-Whitney U-test. 689 (F) Distribution of ranked mean DV model position for the best probability voxel in each 690 predicted glomerulus for all 709 ORs and TAARs for OE zone, OR Class, and gene family. 691 Statistic is Mann-Whitney U-test. 


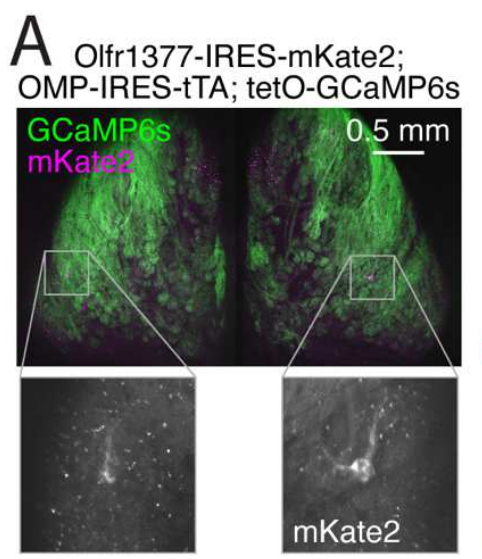

Olfr881-IRES-mKate2; OMP-IRES-tTA; tetO-GCaMP6s
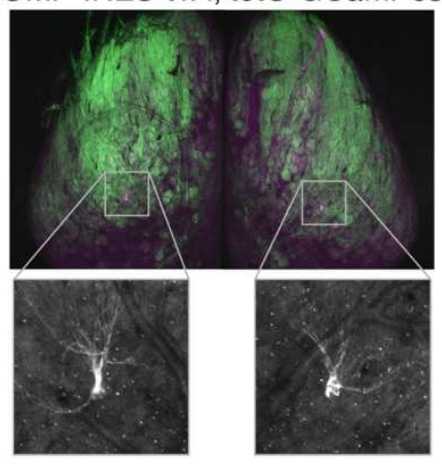

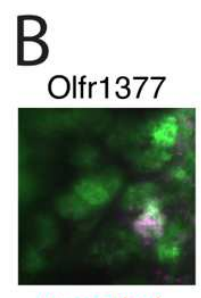

4-methylacetophenone

(0.5)

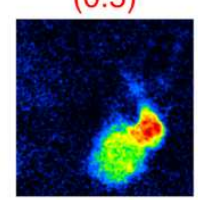

Olfr881

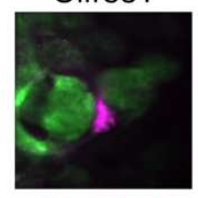

4-methylacetophenone (0.5)

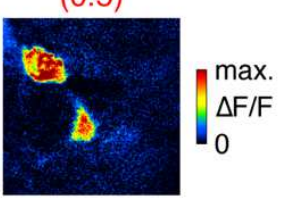

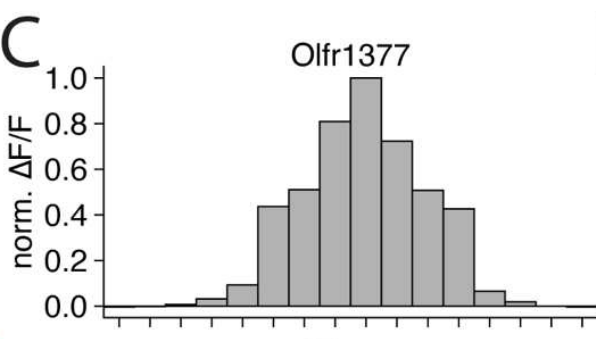

Olfr881
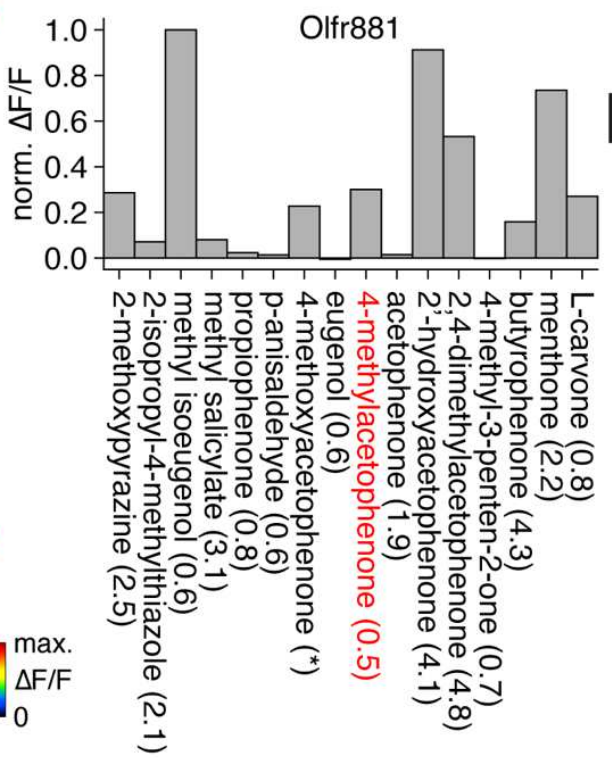

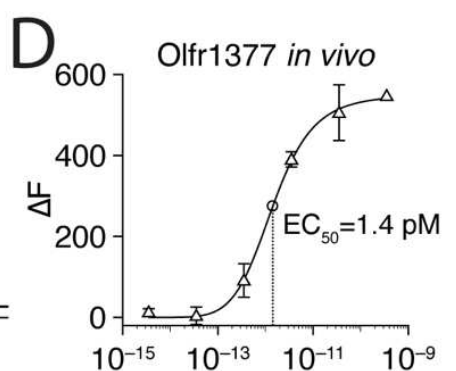

est. concentration (M)

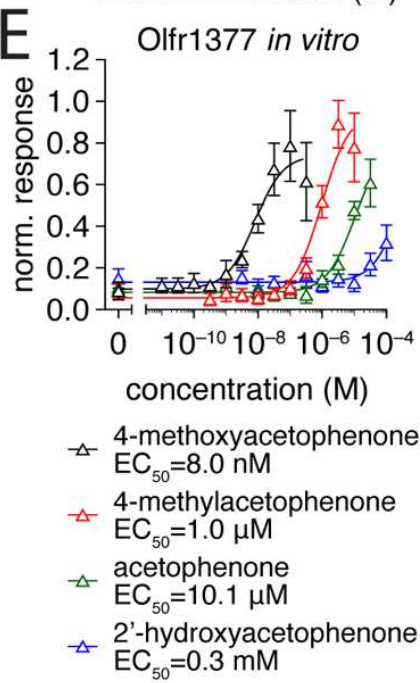

Fig. 6. Deorphanization of Olfr1377 and Olfr881. (A) Whole-mount confocal maximal

694 intensity projection of compound heterozygous Olfr1377-IRES-mKate2; OMP-IRES-tTA; tetO-

695 GCaMP6s and Olfr881-IRES-mKate2; OMP-IRES-tTA; tetO-GCaMP6s mice following two-

696 photon functional imaging. (B) Baseline fluorescence and GCaMP6s $\Delta F / F$ responses to 2-s

697 presentation of 4-methylacetophenone during two-photon functional imaging of the

698 heterozygous lines in (A). Estimated concentration of delivered odorant (in nM) provided in

699 parentheses here and elsewhere. Olfr1377 response map scaled from 0-80\% $\Delta \mathrm{F} / \mathrm{F}$. Olfr881

700 response map scaled from 0-30\% $\Delta F / F$. (C) Spectra of Olfr1377 and Olfr881 glomerular $\Delta F / F$

701 responses (sorted by Olfr1377 response magnitude) to a subset of ligands detected within a

702 larger odorant panel. Odorants presented at an estimated concentration on the order of $10^{\circ}$

$703 \mathrm{nM}$, with the exception of 4-methoxyacetophenone ( 3.5 nM for Olfr881; $\sim 3.5 \times 10^{-3} \mathrm{nM}$ for

704 Olfr1377). (D) In vivo concentration-response function of the Olfr1377 glomerulus to 4- 
705 methoxyacetophenone. (E) In vitro concentration-response function of the Olfr1377 receptor 706 to an array of cyclic ketone odorants. 
SUPPLEMENTARY MATERIALS:

709

710

711

712 713

\section{DATA AVAILABILITY}

All sequencing data will be made publicly available under NCBI BioProject PRJNA773191 upon publication. Additional raw and processed data that support the findings of this study are available from the corresponding authors upon request.

\section{CODE AVAILABILITY}

Key code for the project has been made publicly available at https://github.com/kanazian/obmap.

\section{MATERIALS AND METHODS}

\section{Statistics}

All statistical tests were performed in R. All Mann-Whitney U-tests were two-tailed and performed with continuity corrections using the wilcox.test function from the stats package. Dunn's Krukal Wallis test performed with Benjamini Hochberg false discovery rate correction using the dunn.test function from the dunn.test package. All box and whisker plots display the median value as the middle line, the first and third quartile as the hinges, and the farthest values within 1.5 inter-quartile range of the first or third quartile as the middle line as the whiskers. ${ }^{*}$ indicates $p$ value $\leq 0.05,{ }^{* *}$ indicates $p$ value $\leq 0.01,{ }^{* * *}$ indicates $p$ value $\leq 0.001$.

\section{Animals}

Wild-type C56BL6 mice were purchased from the Jackson Laboratory and used for breeding. Olfr1377-IRES-mKate2 and Olfr881-IRES-mKate2 mice were generated by inserting IRES-mKate2 cassettes downstream of the CDS of each OR using Easi-CRISPR ${ }^{66}$. OMP-IRES-tTA mice ${ }^{67}$ were provided by C. Ron Yu (Stowers Institute, Univ. of Kansas). tetO- 
733 GCaMP6s mice ${ }^{68}$ were purchased from the Jackson Laboratory (Stock No. 024742). Mice

734 were group housed with food and water available ad libitum and kept on a 12-h-light/dark

735 cycle. All procedures for animal handling and tissue collection were approved by the

736 Institutional Animal Care and Use Committees of Duke University and the University of Utah.

737

738

Sample acquisition and sequencing library preparation

Pups were sacrificed between postnatal day 20 and 22 . Whole brains with olfactory

bulbs intact were dissected and placed in a solution of 3\% Low Melting Point Agarose

741 (American Bioanalytical) within an embedding mold (Polysciences Inc, Peel-A-Way R30)

742 before placing on ice. Once solidified, the mold was removed and the agarose-embedded

743 brain was prepared for vibratome sectioning by cutting away agarose to leave a triangular

744 shape with the tip forming at the bulb on the surface that will be cut first for that specific

745 direction. The triangular block was glued (Loctite 404) onto the vibratome stand (Leica

746 VT1000S with Feather carbon blades), submersed in cold 1X HBSS (Gibco) and $100 \mu \mathrm{m}$

747 sections serially cut. Sections were placed into $1.5 \mathrm{~mL}$ tubes and stored at $-80 \mathrm{C}$. $400 \mu \mathrm{L}$ of

748 Buffer RLT (Qiagen) with 10\% BME was added to each frozen tissue section before

749 homogenization at 30,000RPM for 4 seconds using a mounted Biogen PRO200 with 5mm flat

750 tip generator probe. The homogenizer probe was rinsed three times with DI water between

751 samples. RNA was extracted using a Qiagen RNeasy kit according to the manufacturer's

752 instructions. cDNA synthesis was performed using a SMART-Seq v4 (Takara) kit with $10 \mathrm{ng}$

753 total RNA used for input in half-sized reactions. $10 \mathrm{ng}$ cDNA was used in the KAPA Hyperplus

754 library construction kit following the manufacturer's instructions. All quantifications were

755 performed using Qubit.

757 Target capture sequencing and alignment 
We selected a set of ORs, TAARs, V1Rs, and V2Rs for inclusion in our assay based

759

760

761

762

763

764

765

766

767

768

769

770

771

772

773

774

775

776

777

778

779

780

781

782

on the mouse genome annotation available at the time (GRCm38.p4 release M10). This set of transcripts was submitted to Roche Nimblegen for inclusion in a targeted enrichment probe panel. All sections from a single OB were uniquely indexed and combined in equal amounts to create a $1000 \mathrm{ng}$ pool which was processed for target capture according to the manufacturer's protocol using a 20 hour hybridization period. Target capture library pools were sequenced on an Illumina NextSeq 500 Sequencing System in the 75SR or the HiSeq2500 in 50SR mode at the Duke Center for Genome and Biology shared core facility. Snakemake v3.5.5 $5^{80}$ was used to process read files through alignment and quantification. STAR v2.7.0 $\mathrm{d}^{81}$ was used to generate a genome index using the primary assembly and comprehensive gene annotation file of the mouse genome (Gencode GRCm38.p6 release M25). Reads were aligned to this genome index using STAR with default options except for quantMode TranscriptomeSAM which maps genome alignments to transcript coordinates. STAR output transcriptome SAM files were quantified using RSEM v1.3.1 ${ }^{82}$ using default options to generate gene level counts for differential expression.

\section{Sample normalization}

TPM values for each sample from each replicate OB were weighted by a factor that accounts for the proportion of OMP found in each sample and the approximate proportion of the OB glomerular layer collected in the sample (see below: Three-dimensional model of OR glomeruli positions). Weighted TPM was then normalized between 0 and 1 using the minimum and maximum values for each OR. Merged heatmaps were generated using the median normalized values at each position of the expression array for DV and ML heatmaps $(n=3)$. For AP heatmaps, the merged representations was generated from the median of four individual replicates along with the mean value of the four replicates at each position. Matrix 
of median values were then renormalized for each OR across samples. The following ORs

were excluded from all analyses due to abnormally high expression values that potentially 785 indicate ectopic expression of the OR within OB cell types: Olfr287, Olfr32, Olfr361, Olfr1033.

\section{Sequence and position analysis and identification of anterior and posterior OR} associated residues

Mouse OR protein sequences were downloaded from $\mathrm{HORDE}^{83}$ and OR pairwise alignments were computed using the pairwiseAlignment and pid functions from the $\mathrm{R}$ package Biostrings using the BLOSUM62 substitution matrix, a gapOpening penalty of 11 and a 792 gapExtension penalty of 1. 1090 mouse OR protein sequences were aligned using Clustalx 793 with manual adjustments as previously published ${ }^{65}$. To identify residues that were more conserved by the 139 ( $30 \%$ set) most anterior, ventral, Class II ORs than by chance, we 795 simulated distributions of Grantham distances for random subsets of 70 ( $50 \%$ of size of set) ventral Class II ORs for all pairwise combinations for each residue in the alignment. Random 797 sampling was performed 1000 times. The per-residue mean Grantham distances were computed for all combinations of the 139 most anterior, ventral, Class II ORs and used to find p-values under the null distribution after FDR correction. Alignment positions with gaps in more than $10 \%$ of OR sequences were excluded from the analysis. Analysis was repeated for 801 all sets $(20 \%, 27.5 \%, 35 \%)$ for both anterior and posterior Class II ORs. Visualization of the 802 803 804 structural sequence for Olfr539 were generated using GPCRsnakeplotter (https://github.com/Yue-Jiang/snakeplotter). Sequence logos were created using WebLogo (http://weblogo.threeplusone.com/) using default settings. 805 
The protocol follows a previously published method ${ }^{57}$. Aligned protein sequences of

8081092 mouse ORs were manually aligned to pre-aligned protein sequences of 11 GPCRS 809 including bovine rhodopsin (PDB ID 1U19), human chemokine receptors CXCR4 (PDB ID 810 3ODU) and CXCR1 (PDB ID 2LNL), and human adenosine a2A receptor (PDB ID 2YDV) 811 using Jalview ${ }^{84}$. Four experimental GPCR structures (1U19, 3ODU, 2YDV, and 2LNL) were 812 used as templates to build the Class II mouse consensus OR by homology modeling using 813 Modeller. Five models were obtained and the one fulfilling several constraints (sufficiently 814 large binding cavity, no large folded structure in extracellular loops, all TMs folded as $\alpha-$ 815 helices, and a small a-helix structure between TM3 and TM4) was kept for further 816 visualization.

\section{Differential expression analysis}

The R package, edgeR (v3.34.0), was applied to the sample count table for all genes

820 in the reference ${ }^{85}$. Common, trended, and tagwise dispersions were estimated separately and 821 in the sequence listed. For identification of ORs in the functional imaging surface and ORs 822 responding to 4-methylacetophenone, results were subset to olfactory receptor genes 823 (pseudogenes included) and FDR values were recalculated from the $p$ values obtained by 824 performing differential expression using all genes.

\section{Three-dimensional model of OR glomeruli positions}

828 Blender. An array of cubes with the number of cubes per dimension matching the number of 829 sections collected from each dimension was stretched in each dimension to encompass the 830 entire $\mathrm{OB}$ in order to account for difference in age of mice. OB and cube structures were 831 exported as separate .ply files and imported into $\mathrm{R}$ where a custom script was used to 
832 determine the position of cubes which contain OB surface polygons. Additional OB surface-

833 containing cubes were included to remove gaps, model the medial surface, and account for a

$834100 \mu \mathrm{m}$ wide glomerular layer within the outer surface of the OB. The final set of OB surface-

835 containing cubes was exported as three-dimensional coordinates for use as a scaffold for the 836 shape of the OB glomerular layer.

837 The composition of glomeruli in each voxel along the OB surface was calculated as the 838 weighted average of the composition obtained from sequencing each OB section that 839 intersected that voxel weighted by the total number of surface voxels in each section. To 840 account for sequencing noise, the composition of glomeruli in each sequence was estimated 841 using a Bayesian multinomial-Dirichlet model with a Dirichlet prior parameter of 0.65 reflecting 842 the weak prior knowledge that each section likely contained only a subset of all possible 843 glomeruli in the OB. For each sequenced section, 1000 posterior samples from this model 844 were obtained and used in subsequent calculations of the glomerular composition in each

845 surface voxel. Custom functions were applied to determine positional clusters of high 846 probability voxels for each gene based on the Bayesian posterior probability and the expected

847 DV location of the OR based on class and Tan and Xie Chem. Senses. 2018 index identity.

848 Briefly, each gene was designated as dorsal, ventral, unusual zone, or non-OR based on

849 gene name, OR class, OE position, and OB mean position. Based on this DV designation, the 850 highest probability voxel cluster (minimum 3 adjacent voxels) from the corresponding DV 851 hemisphere on each side of the line of symmetry was picked. The probability of the medial 852 and lateral clusters was then compared and used to define the region of mirror symmetry for 853 the cluster with the lower probability with the lower probability cluster being repicked from a 854 mirror symmetry field across the line of symmetry. If the medial cluster had a higher 855 probability, the lateral cluster was repicked from a field that was from the lateral half and 856 ranging from 6 sections anterior to 1 section posterior of the medial cluster. If the lateral 
cluster had a higher probability, the medial cluster was repicked from a field that was from the

858 medial half and ranging from 6 sections posterior to 1 section anterior of the lateral cluster.

859 Clusters that were found within 6 sections of the front or back of the model were repicked

860 from fields between the end of the model and 6 sections inward. The probability of these two

861 clusters was then compared to that of a single larger ( 5 adjacent voxels) cluster picked from a

862 band of voxels running adjacent to the line of symmetry in order to process ORs whose

863 proximity to the symmetry line has led to a single glomerulus or closely positioned glomeruli. If

864 the probability of this symmetry line voxel cluster was greater than that of the best probability

865 of the medial or lateral glomeruli, the symmetry line voxel cluster was chosen as the predicted 866 position. If not, the split glomeruli were selected as the predicted position. ORs classified as

867 being in the unusual zone in the OE were predicted as a single large cluster within the 868 anterior, ventral, central face of the OB. Non-OR genes were predicted using the same 869 method as dorsal ORs with only the top probability voxel cluster being returned.

Comparison against ORs with known glomeruli positions was performed by superimposing images of mapped positions from publications on a blank OB 3D model and recording AP, DV, ML coordinates for the boundaries of the depicted glomerulus. For each glomerulus of each OR with known information, we calculated all pairwise three-dimensional distances between the voxels within the predicted position and the depicted positions.

875 Reported value was the minimum distance observed with any overlap between predicted and depicted position resulting in a value of 0 .

pS6-IP RNA-Seq

Three P21 mice of either sex were used per odorant or control condition. Animals were individually habituated in sealed containers for $1 \mathrm{~h}$, then transferred to a new container containing a piece of filter paper enclosed in a cassette (Tissue-Tek) spotted with $10 \mu \mathrm{l}$ of 4- 
methylacetophenone diluted at $1 \%(\mathrm{v} / \mathrm{v})$ in distilled water or with $10 \mu \mathrm{l}$ of distilled water (no

883 odor control). After $1 \mathrm{hr}$ of exposure, the animals were euthanized, then had their olfactory

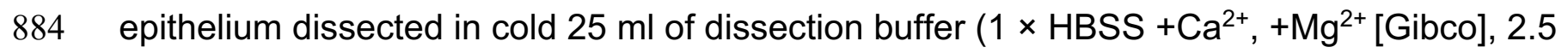
$885 \mathrm{mM}$ HEPES pH 7.4, $35 \mathrm{mM}$ glucose, $100 \mu \mathrm{g} / \mathrm{ml}$ cycloheximide, $5 \mathrm{mM}$ sodium fluoride, $1 \mathrm{mM}$ 886 sodium orthovanadate, $1 \mathrm{mM}$ sodium pyrophosphate, $1 \mathrm{mM}$ beta-glycerophosphate). The 887 dissected tissues were transferred to $1.35 \mathrm{ml}$ of cold homogenization buffer ( $150 \mathrm{mM} \mathrm{KCl}, 5$ $888 \mathrm{mM} \mathrm{MgCl}$, $10 \mathrm{mM}$ HEPES pH 7.4, $100 \mathrm{nM}$ Calyculin A, $2 \mathrm{mM}$ DTT, $100 \mathrm{U} / \mathrm{ml}$ RNasin 889 [Promega], $100 \mu \mathrm{g} / \mathrm{ml}$ cycloheximide, $5 \mathrm{mM}$ sodium fluoride, $1 \mathrm{mM}$ sodium orthovanadate, 1 $890 \mathrm{mM}$ sodium pyrophosphate, $1 \mathrm{mM}$ beta-glycerophosphate, 1x protease inhibitor [Roche]). 891 Bones were removed, and the tissue was mechanically dispersed three times at $250 \mathrm{rpm}$ and 892 nine times at $750 \mathrm{rpm}$ using a homogenizer (Glas-Col). The homogenates were centrifuged at $8934600 \mathrm{rpm}$ for $10 \mathrm{~min}$ at $4^{\circ} \mathrm{C}$ in a $1.5 \mathrm{ml}$ Lobind tube (Eppendorf), then the supernatants were 894 collected into a new $1.5 \mathrm{ml}$ Lobind tube and combined with a mixture of $90 \mu \mathrm{l} 10 \%$ NP-40 and $89590 \mu \mathrm{l} 300 \mathrm{mM}$ DHPC (Avanti Polar Lipids). The samples were centrifuged at $13000 \mathrm{rpm}$ for 10 $896 \mathrm{~min}$ at $4^{\circ} \mathrm{C}$, then the supernatants were collected into a new $1.5 \mathrm{ml}$ Lobind tube and incubated 897 with $20 \mu \mathrm{l} \mathrm{pS6}$ antibody (Cell Signaling \#5364) for $1.5 \mathrm{hr}$ at $4^{\circ} \mathrm{C}$ under constant rotation. 898 During the last 30 minutes of incubation, $100 \mu \mathrm{l}$ Protein A Dynabeads (Invitrogen) were 899 prepared by washing three times with $900 \mu \mathrm{l}$ of wash buffer $1(150 \mathrm{mM} \mathrm{KCl}, 5 \mathrm{mM} \mathrm{MgCl} 2,10$ $900 \mathrm{mM}$ HEPES $\mathrm{pH} 7.4,0.05 \% \mathrm{BSA}, 1 \% \mathrm{NP}-40)$. The samples were added to the beads and 901 incubated under constant rotation for $1 \mathrm{hr}$ at $4^{\circ} \mathrm{C}$, followed by four washes with $700 \mu \mathrm{l}$ of wash 902 buffer 2 (350 mM KCl, 5 mM MgCl 2,10 mM HEPES pH 7.4, 1\% NP-40, 2 mM DTT, 100 U/ml 903 recombinant RNasin [Promega], $100 \mu \mathrm{g} / \mathrm{ml}$ cycloheximide, $5 \mathrm{mM}$ sodium fluoride, $1 \mathrm{mM}$ 904 sodium orthovanadate, $1 \mathrm{mM}$ sodium pyrophosphate, $1 \mathrm{mM}$ beta-glycerophosphate). The 905 samples were moved to room temperature at the final wash, and the elution was performed 
with $350 \mu$ l Buffer RLT (Qiagen). The RNA present in the eluate was purified with the RNeasy 907 Micro kit (Qiagen).

908

Heterologous cell expression

The Dual-Glo luciferase assay was performed as described previously ${ }^{84}$. Briefly, 911 Hana3A cells ${ }^{86,87}$ were plated on 96 -well plates in MEM supplemented with $10 \%$ FBS. After $91218-24 \mathrm{hr}$ incubation at $37^{\circ} \mathrm{C}$ and $5 \% \mathrm{CO}_{2}$, cells were transfected with $100 \mathrm{ng} / \mu \mathrm{L}$ plasmids 913 coding for Olfr1377 (5 $\mu \mathrm{L} /$ plate), muscarinic acetylcholine receptor M3R (2.5 $\mu \mathrm{L} / \mathrm{plate})$, 914 RTP1S (5 $\mu \mathrm{L} /$ plate), pRL-SV40 (5 $\mu \mathrm{L} /$ plate) and CRE-luciferase (10 $\mu \mathrm{L} /$ plate $)$ using 915 Lipofectamine 2000 (Invitrogen). pCl vector (5 $\mu \mathrm{L} /$ plate) was used instead of Olfr1377 plasmid 916 for the transfection of control cells. 18-24 $\mathrm{h}$ after the transfection, the culture medium was 917 replaced with serial dilutions of the odorants in CD293 medium (GIBCO), then the plates were 918 incubated for 3.5 hours at $37^{\circ} \mathrm{C}$ and $5 \% \mathrm{CO}_{2}$ without the lid. Luminescence was measured 919 using a Polarstar Optima plate reader (BMG). Luminescence values (LV) were determined by 920 subtracting basal luminescence (of an empty 96-well plate), then dividing firefly luminescence 921 by renilla luminescence (to control for transfection efficiency and cell viability) as in the 922 formula: LV = (firefly luminescence - 400)/(Renilla - 400). The LV values were normalized and 923 plotted using GraphPad Prism 9 by establishing the minimum LV $=0$ and the maximum LV = 9241 and using the analysis log(agonist) vs. response (three parameters). CAS numbers for 925 tested odorants are as follows: acetophenone - 98-86-2, 2-hydroxyacetophenone - 118-93-4, 926 4-methylacetophenone - 122-00-9, 4-methoxyacetophenone - 100-06-1.

\section{Functional imaging} Adult mice (2-3 months old) of both sexes were anesthetized and prepared for 
previously described ${ }^{88}$. Imaging was conducted using a resonant-scanning two-photon

932 microscope (Sutter Instruments) coupled to a pulsed Ti:Sapphire laser (Mai Tai HP, Spectra 933 Physics) tuned to $920 \mathrm{~nm}$ and a Fidelity-2 $1070 \mathrm{~nm}$ laser (Coherent) and equipped with a 16X

9340.8 N.A. water-immersion objective (Nikon), with emission separated by green $(520 / 65 \mathrm{~nm})$ 935 and red $(641 / 75 \mathrm{~nm})$ filters and collected by GaAsP photomultipliers (Hamamatsu H10770B).

936 Glomeruli were imaged at $15.5 \mathrm{~Hz}$ through thinned-bone windows. Odorants were presented 937 using a custom olfactometer ${ }^{88}$ in pseudorandom order (except for 4-methoxyacetophenone 938 concentration series), with $2 \mathrm{~s}$ delivery duration and variable inter-delivery intervals. Odorants 939 were diluted in caprylic/capric medium chain triglycerides (C3465, Spectrum Chemical Mfg. 940 Corp.).

941 For response maps and spectra (Fig. $6 \mathrm{~B}$ and $\mathrm{C}), \Delta \mathrm{F} / \mathrm{F}$ responses were calculated as 942 the difference in mean fluorescence within $4 \mathrm{~s}$ following odorant onset from the $4 \mathrm{~s}$ preceding 943 odorant onset (the baseline fluorescence), divided by the baseline fluorescence. For display, $944 \Delta \mathrm{F} / \mathrm{F}$ response maps were bilinearly interpolated by a factor of 2 and low-pass filtered 945 (Gaussian, standard deviation $=1$ pixel) to improve map resolution and reduce map noise, 946 respectively. For the concentration-response function of the Olfr1377 glomerulus to 4947 methoxyacetophenone (Fig. $6 \mathrm{D}$ ), $\Delta \mathrm{F}$ responses were calculated as the difference in mean 948 fluorescence within $2.5 \mathrm{~s}$ following odorant onset from the baseline fluorescence. For the 949 lowest three odorant concentrations (in which responses fully decayed before subsequent 950 odorant presentations), baseline fluorescence was calculated as the mean fluorescence 951 within the $1 \mathrm{~s}$ preceding odorant onset. For the highest three odorant concentrations (in which 952 responses failed to fully decay before subsequent odorant presentation), $\Delta \mathrm{F}$ responses were 953 calculated using the baseline fluorescence observed during the final $3.5 \times 10^{-13} \mathrm{M}$ odorant 954 presentation. All $\Delta \mathrm{F} / \mathrm{F}$ and $\Delta \mathrm{F}$ responses are averaged across three odorant presentations. 955 All analysis was performed using custom code written in MATLAB (MathWorks). 
957 Whole-mount visualization

958 Adult mice of both sexes were transcardially-perfused with PBS followed by $4 \%$

959 paraformaldehyde and postfixed overnight before brains were extracted. For whole-mount

960 confocal imaging, the ventral surface of the brain was adhered to a $35 \mathrm{~mm}$ culture dish to limit

961 movement, covered in PBS to maintain tissue hydration, and $10 \mu \mathrm{m}$ step z-stacks collected

962 using a FluoView FV1000 (Olympus) confocal microscope equipped with 5X air objective

963 (Olympus). Image analysis and maximal intensity projections were performed in ImageJ. For

964 mapping glomerular position from whole-mount preparations, OBs from postfixed brains were

965 removed intact and epifluorescence images of the dorsal and, subsequently, medial surfaces

966 of each OB captured at 5x magnification, with each surface positioned roughly perpendicular

967 to the imaging axis. Images were stitched manually in Photoshop before registration of OBs

968 and glomerular position mapping.

969

970

971 

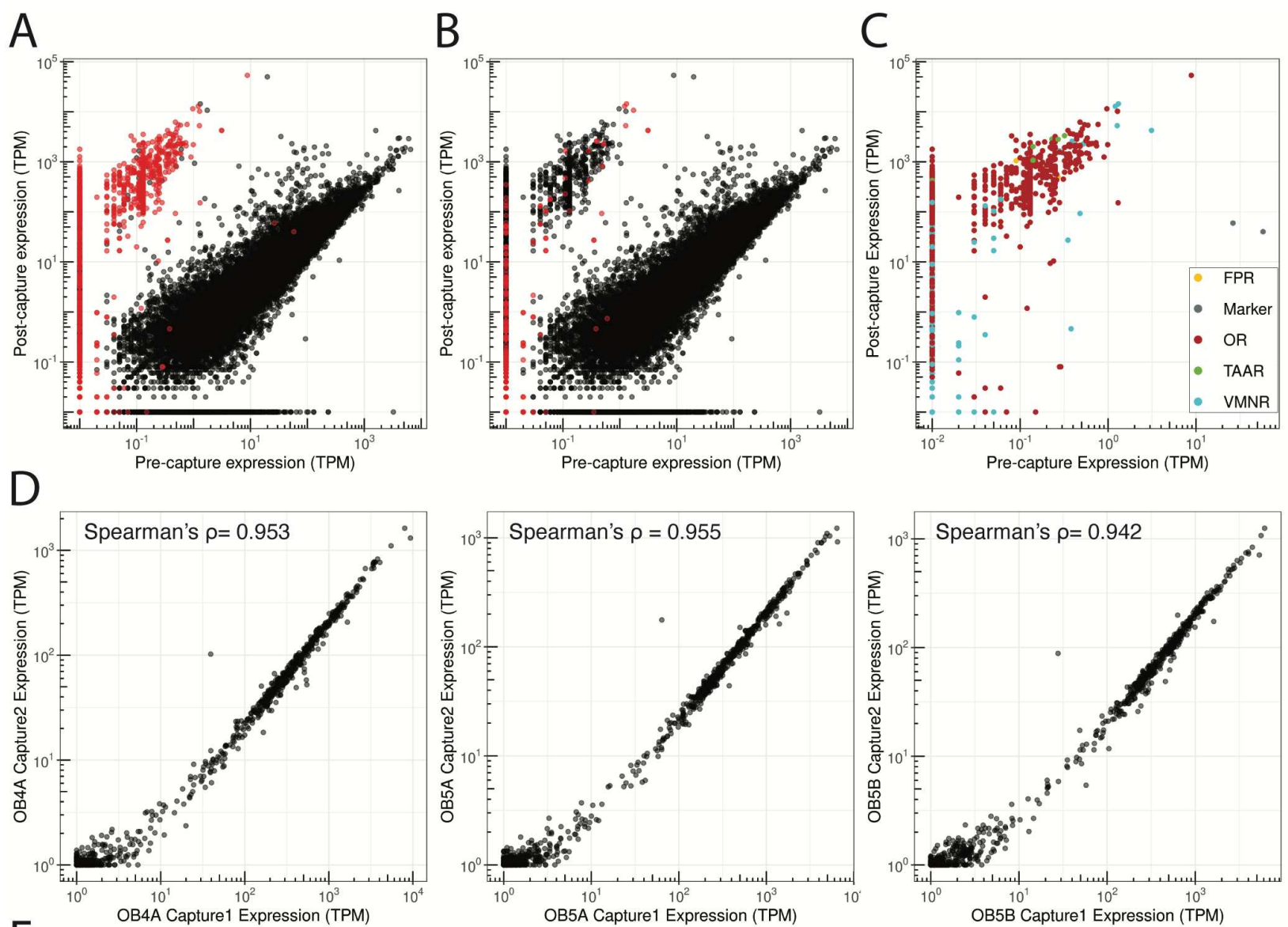

$\mathrm{E}$
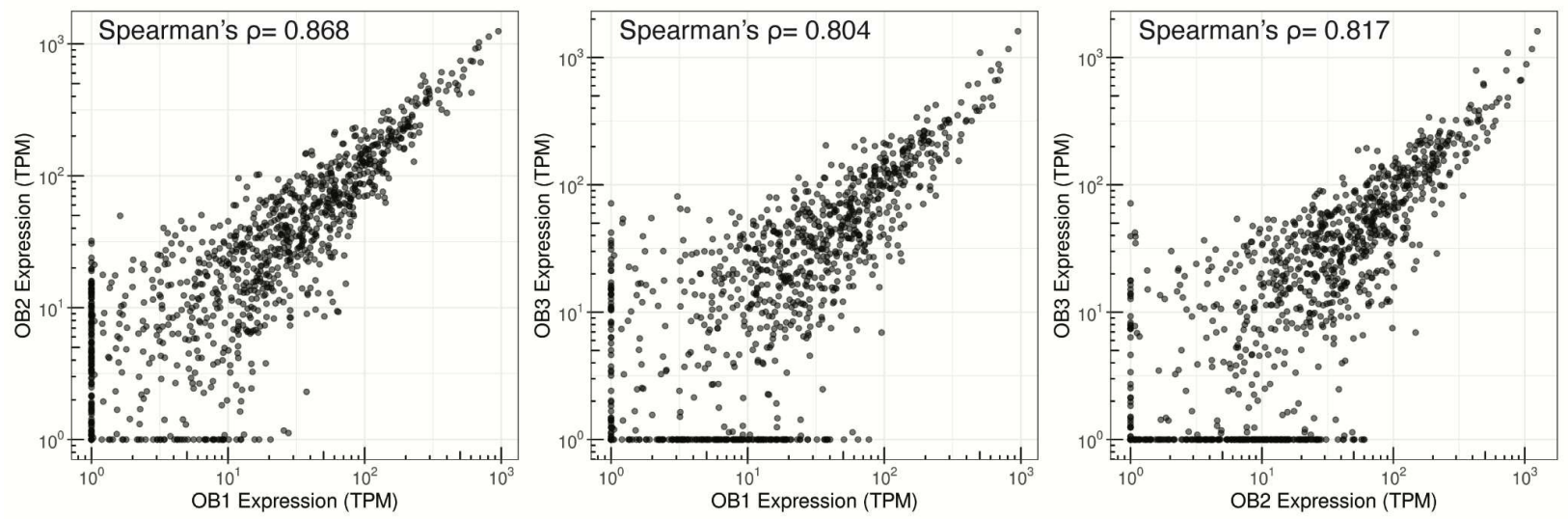

973 Fig. S1. Targeted capture sequencing is consistent for enrichment of OR genes. (A)

974 Pre- and post-capture normalized abundances of intact probed genes (red) and intact non-

975 probed genes (black) from a whole OB. (B) Pre- and post-capture normalized abundances of

976 intact vomeronasal (Vmnr) genes (red) and intact non-Vmnr genes (black) from a whole OB.

977 (C) Pre- and post-capture normalized abundances of target gene families. (D) Technical 
978 replicates of OR and TAAR gene abundances from independent capture enrichments using 979 two different whole-OB RNA samples (OB4, OB5) combined with different ERCC spike-in 980 mixtures (A, B). (E) Biological replicates of OR and TAAR gene abundances from 981 independent capture enrichments of three different whole-OB samples (OB1, OB2, OB3). 


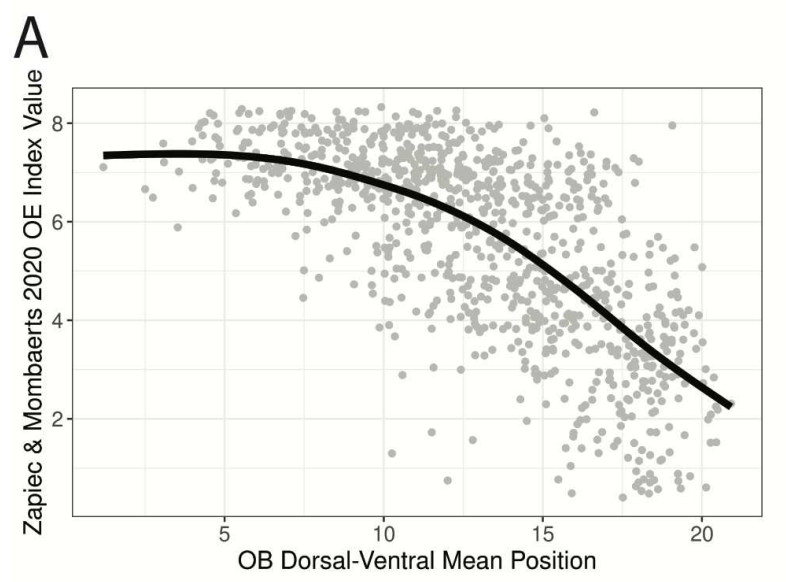

983 Fig. S2. Dorsoventral OB spatial sections correlate with known OE positions. (A) Loess 984 smoothed regression of OE DV index from Zapiec and Mombaerts, Cell. Reps. 2020 across 985 DV mean positions from our targeted spatial data. 


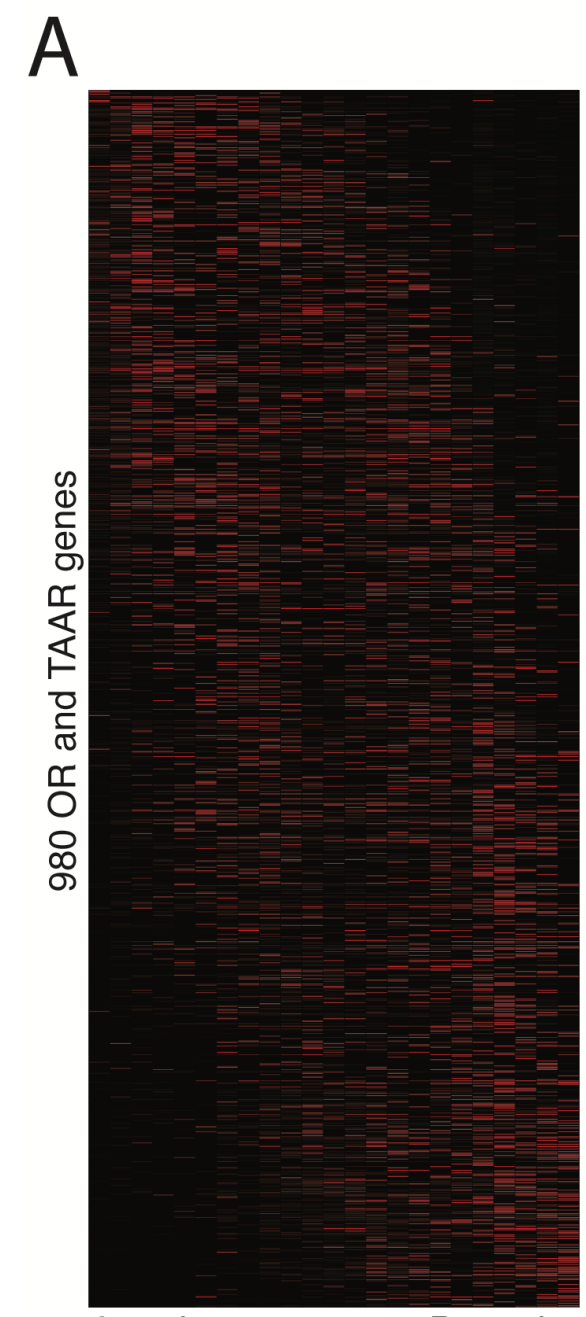

Anterior

Posterior

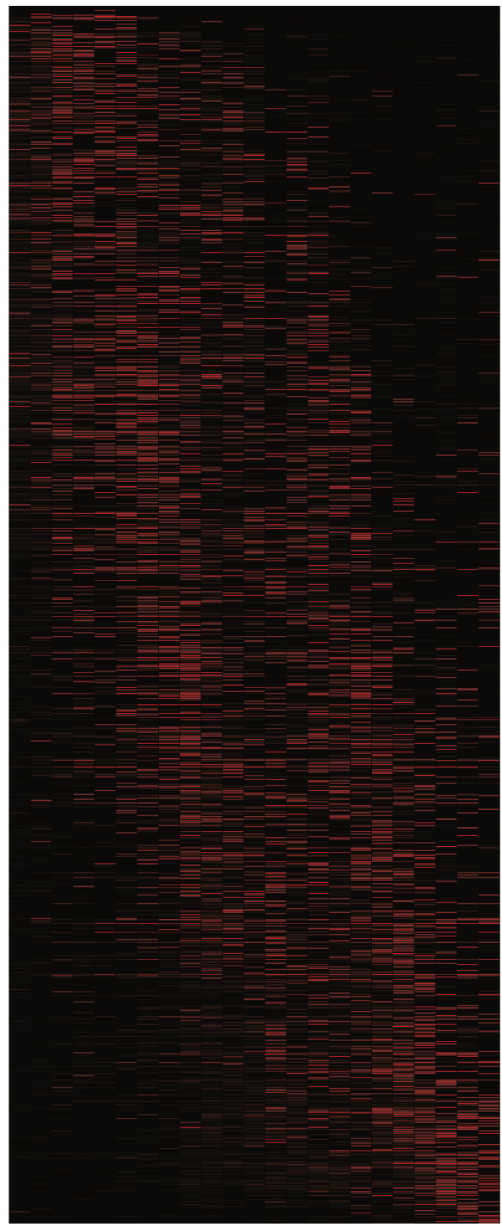

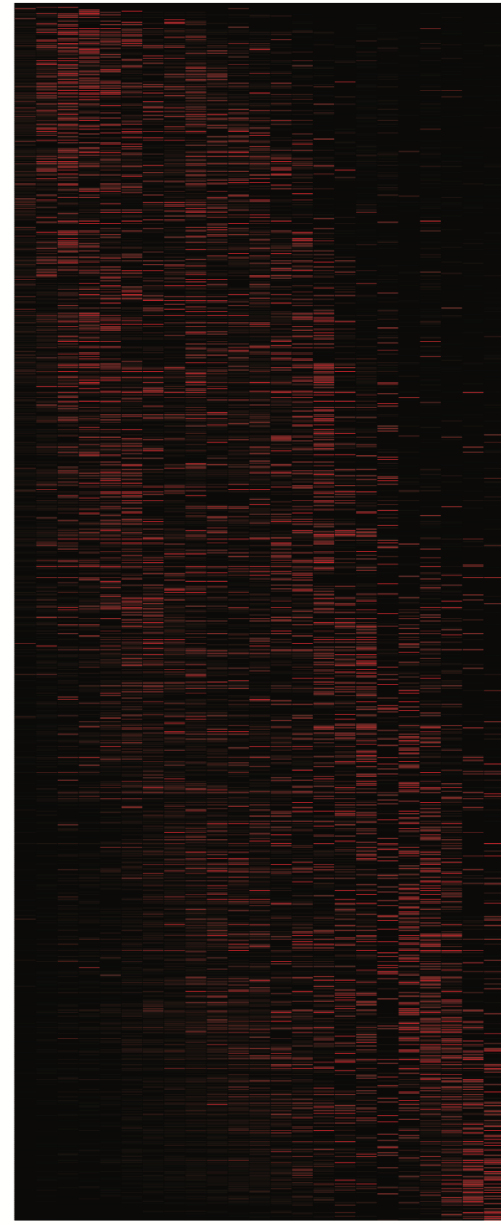

Normalized TPM

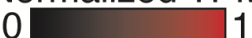

986

987 Fig. S3. ORs display bimodal distributions along the anteroposterior axis. (A) Heatmaps

988 for 980 ORs and TAARs across 23 AP sections sorted by mean position of expression from

989 three additional replicate mice. 

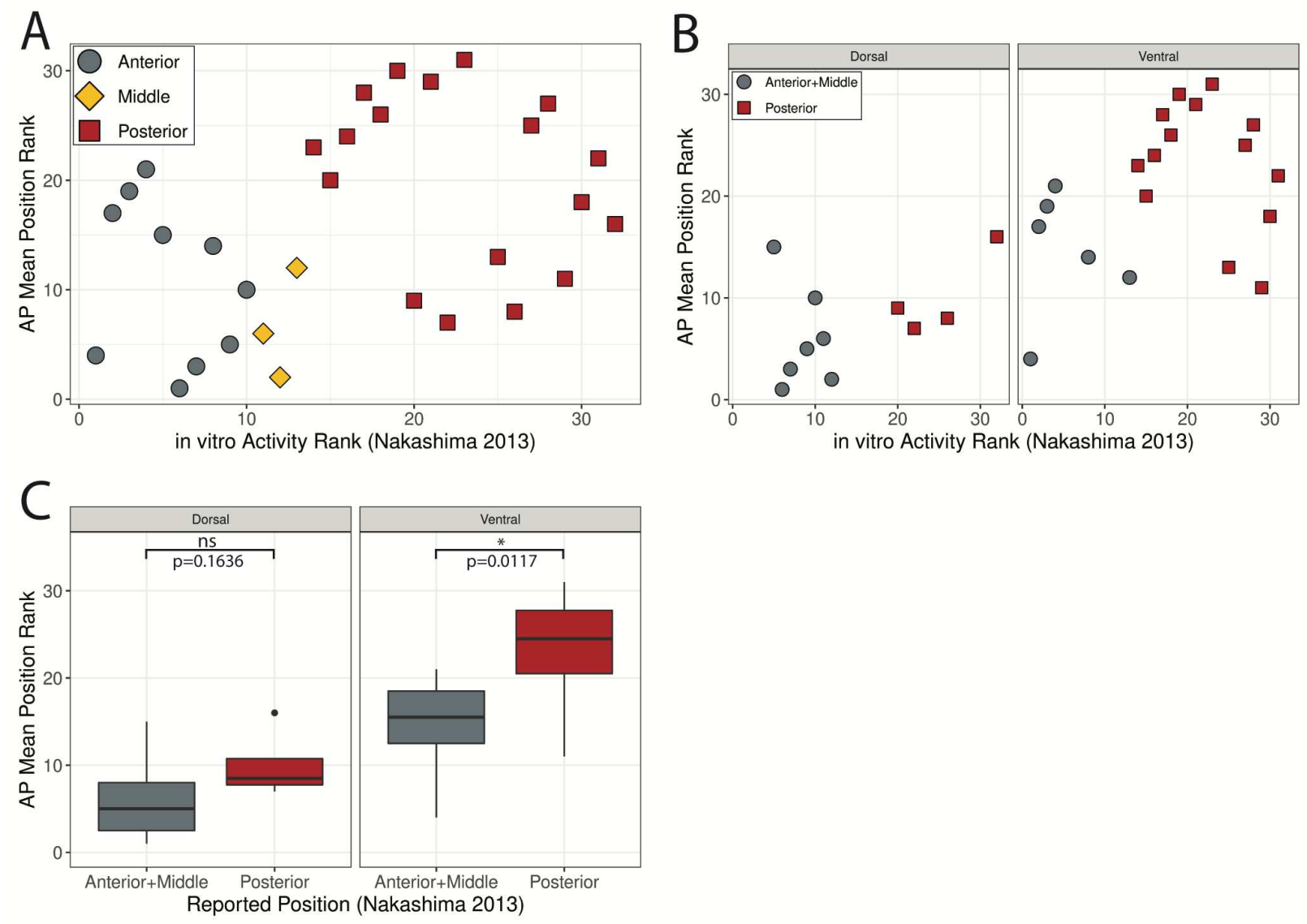

991 Fig. S4. Mapped AP positions are consistent with published AP data. (A) Scatter plot of

99231 ORs present in our AP dataset examining the relationship between ranked mean AP

993 position and in vitro activity rank from Nakashima et al. Cell. 2013. Color indicates the

994 reported OB position from which the OR was cloned from. (B) Data from B separated by OE

995 DV zone. (C) Data from B, grouped. Statistic is Mann-Whitney U-test. 

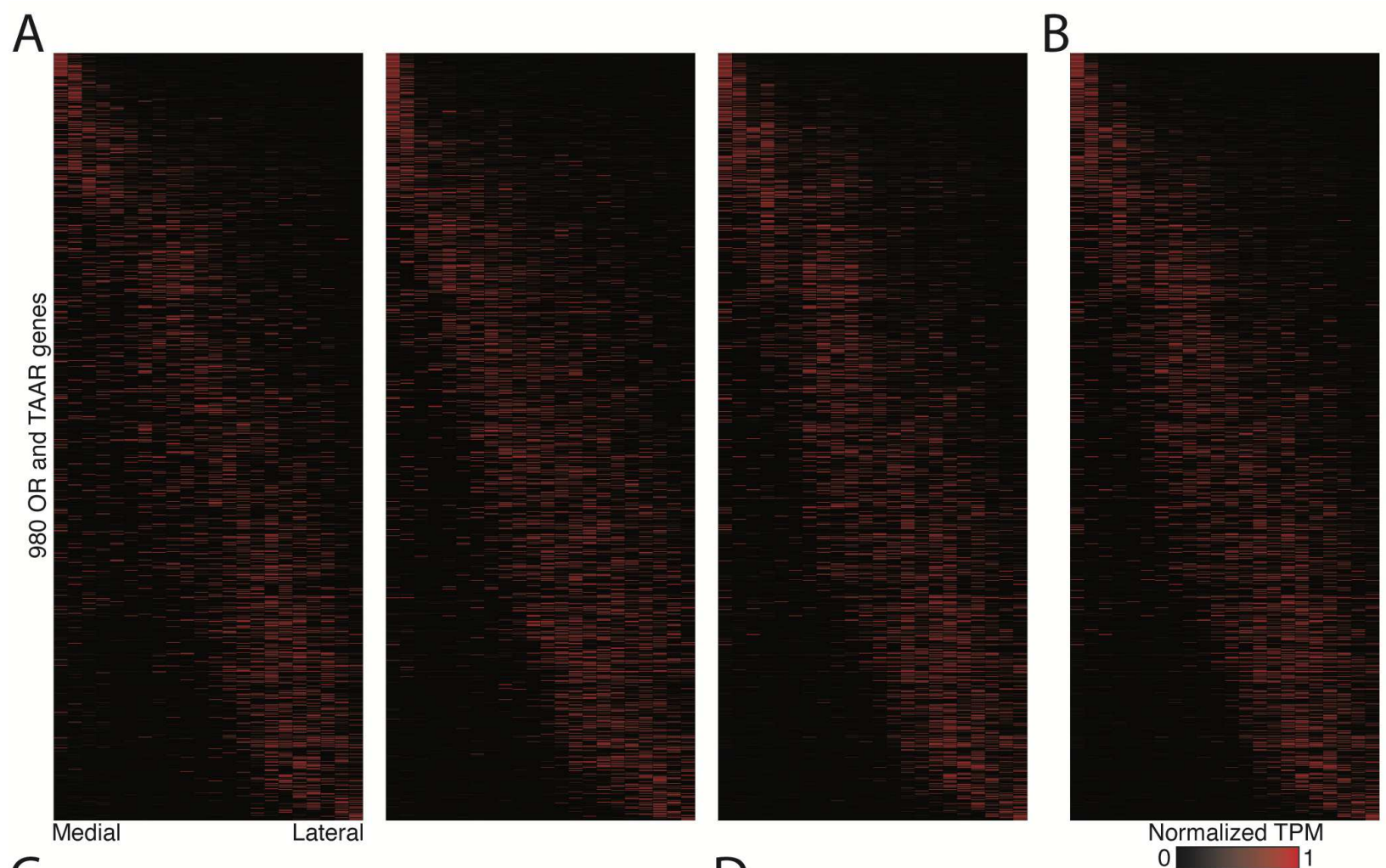

C
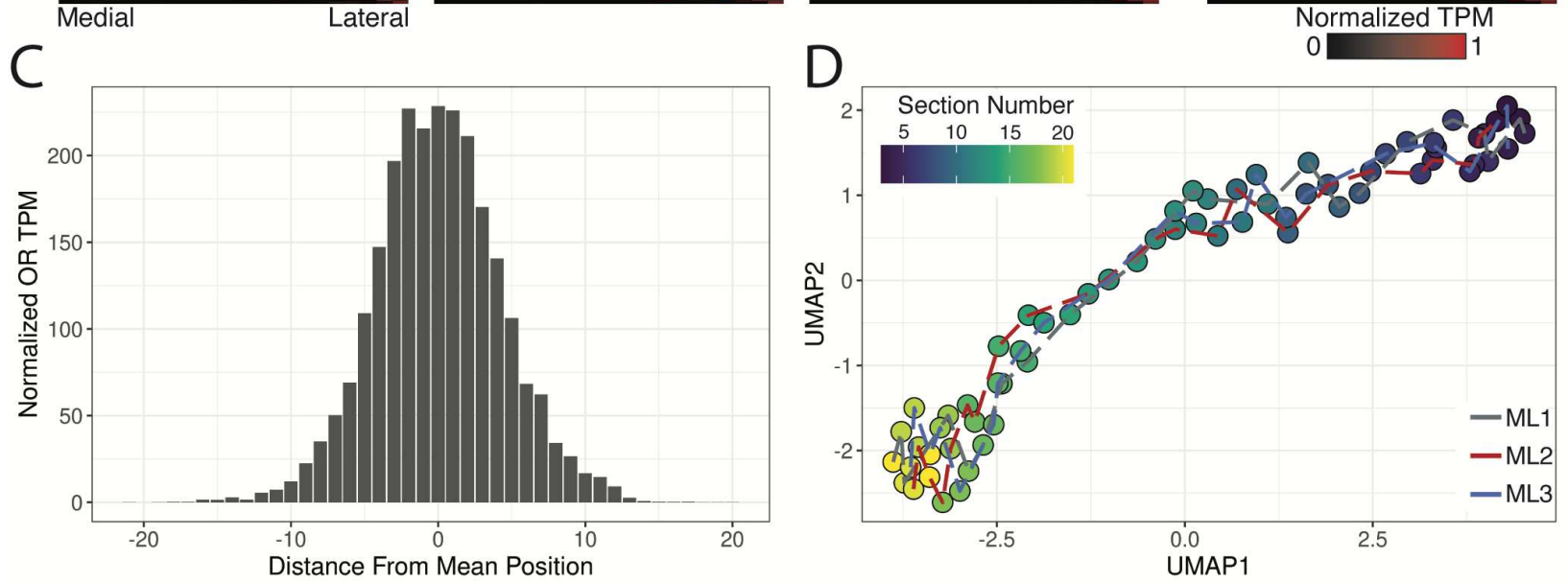
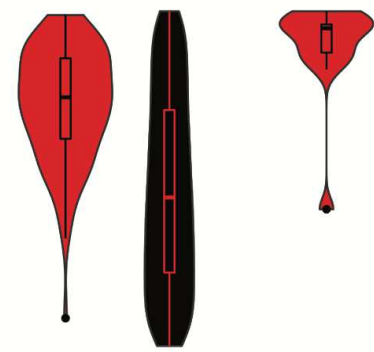

TAAR

Gene Family $\mathrm{p}=2.666 \mathrm{e}-07$ $n=14 \quad n=966$

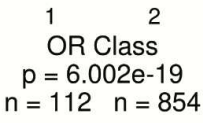

.

Medial OB

mean position

Lateral OB

mean position

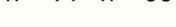


999 representation of $\mathrm{A}$. Order of $\mathrm{Y}$-axis genes is consistent across all heatmaps. (C) Distribution .000 of normalized TPM (maximum observed value $=1$, minimum observed value $=0$ ) for all 980 001 ORs and TAARs from position of mean expression. (D) UMAP projection of $66 \mathrm{ML}$ samples 002 from all three replicates. (E) Distribution of ranked ML mean positions for the 980 ORs and 003 TAARs by OR class and gene family. Statistic is Mann-Whitney U-test. 

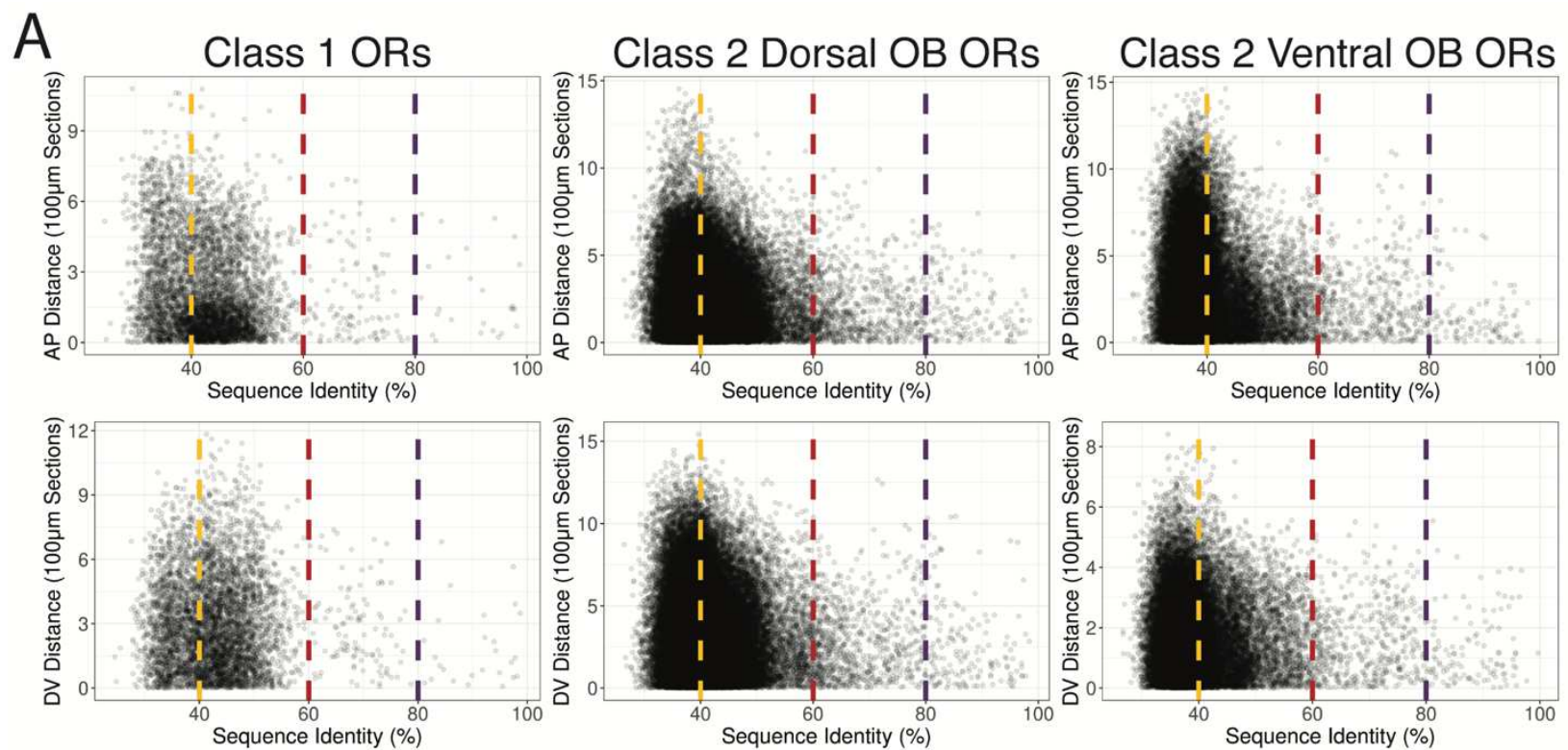

004
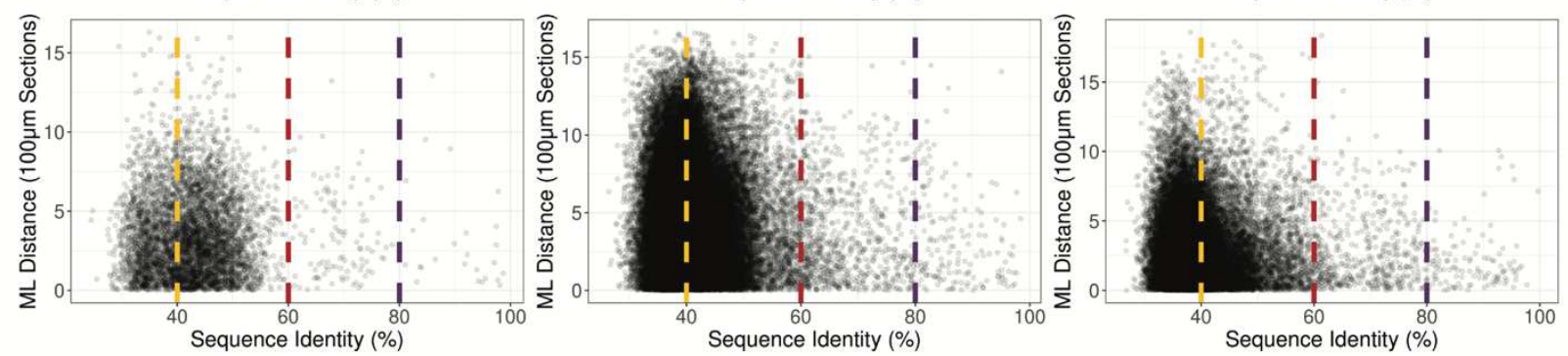

005 Fig. S6. Relationship of OR protein sequence and OB position. (A) Scatter plot of mean

006 position distance and OR protein sequence identity for ORs. Dashed lines indicate family

007 (40\% in yellow), subfamily (60\% in red), and highly similar ( $80 \%$ in magenta) OR level 
008 homology cutoffs for the AP (top), DV (middle), and ML dimensions (bottom).

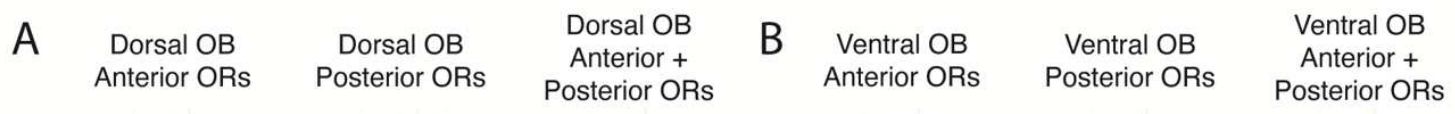

Set 1:

$20 \%$ most A/P
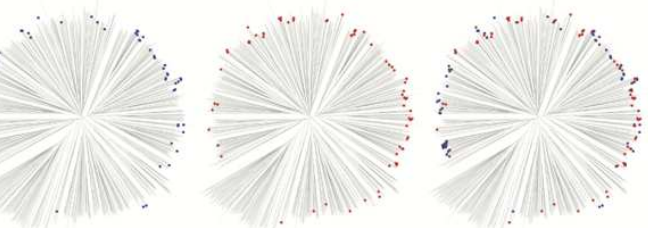

Set 2:

$27.5 \%$ most A/P
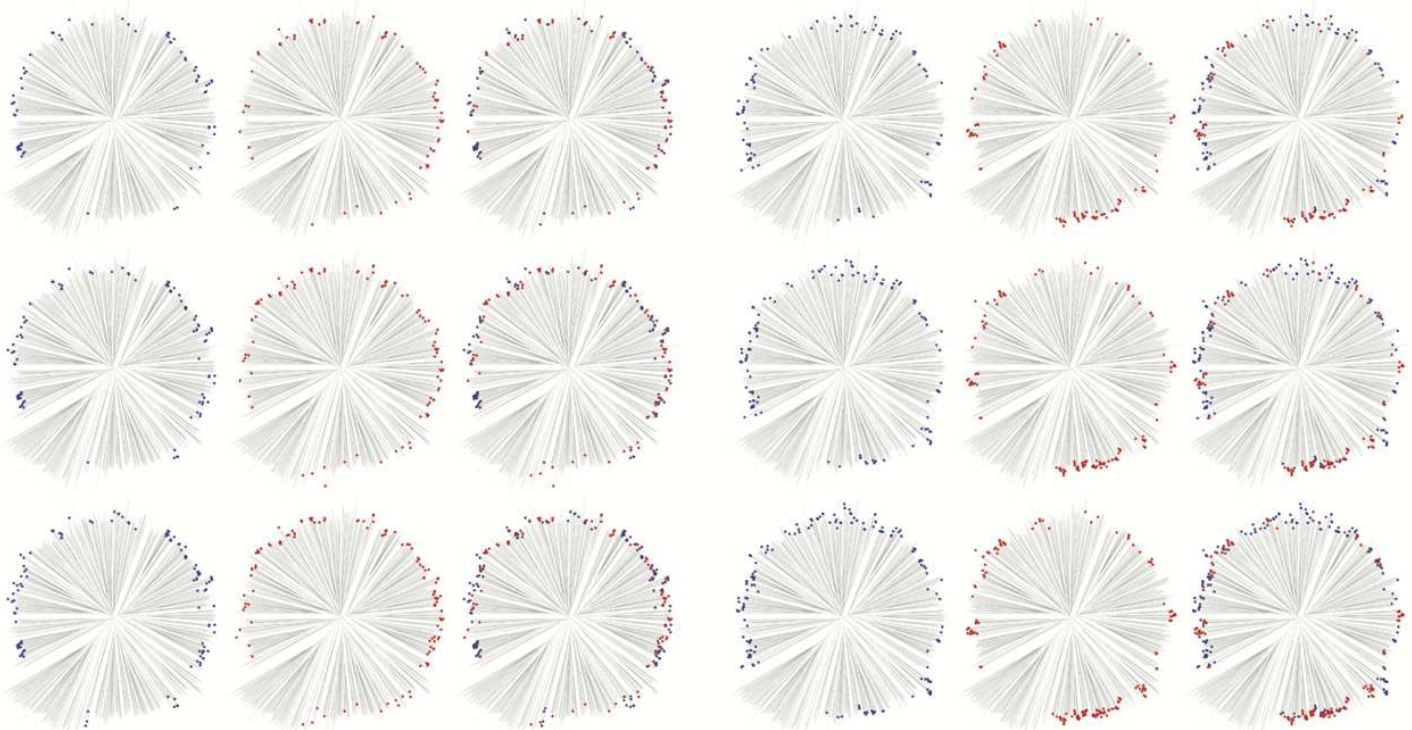

Set 3:

$35 \%$ most $A / P$
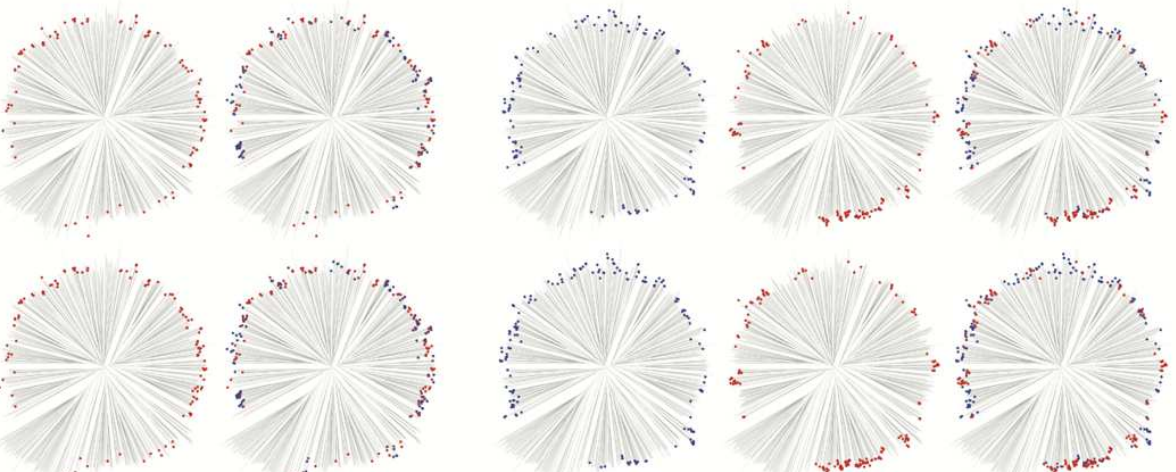

C
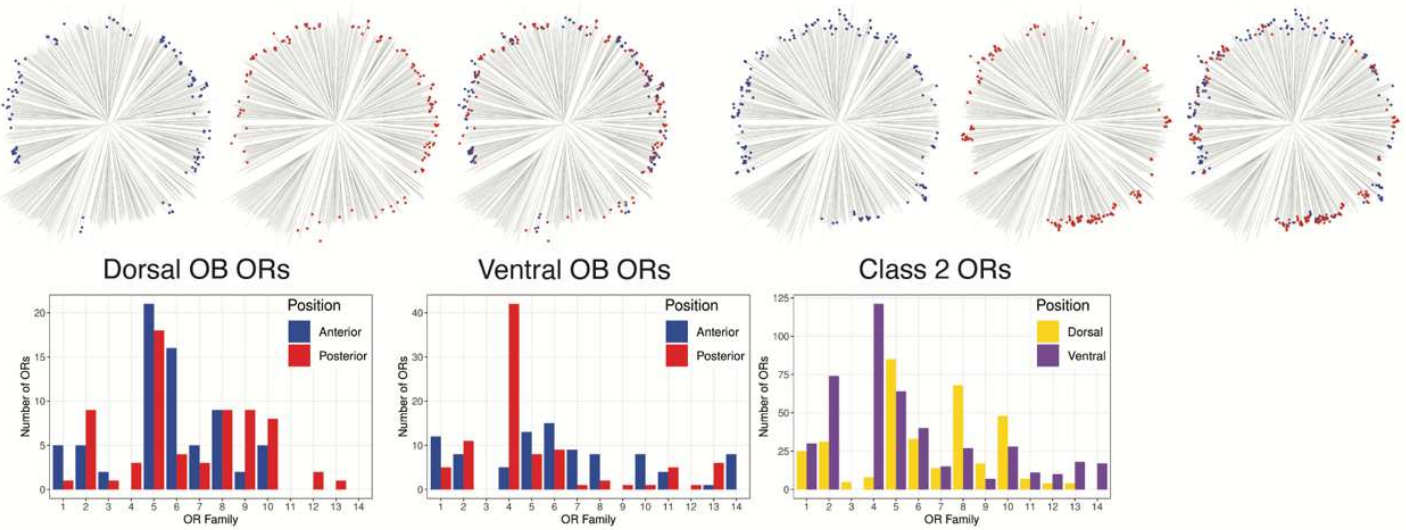

Set 1:

$20 \%$ most A/P
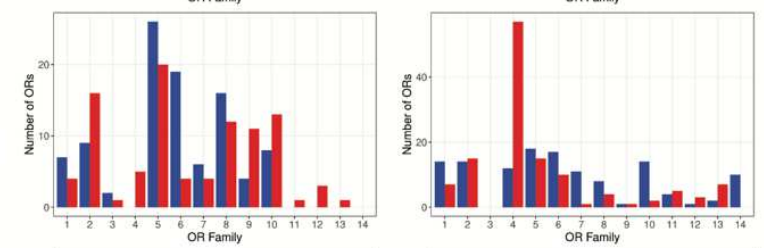

Set 2:

$27.5 \%$ most $\mathrm{A} / \mathrm{P}$
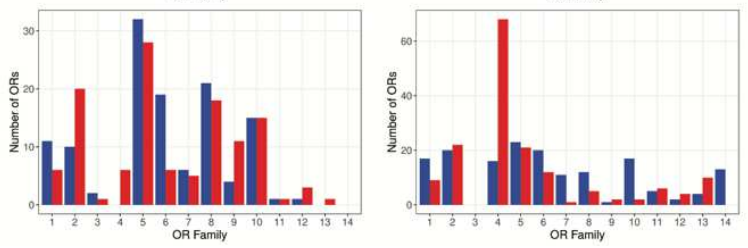

010 Fig. S7. Anterior and posterior ORs along the dorsal and ventral OB. (A) Phylogenetic

011 trees for anterior sets of Class II dorsal OB ORs (blue, left), posterior sets of Class II dorsal

012 OB ORs (red, middle), and combined sets of dorsal anterior and posterior Class II OB ORs

013 (right). Sets of different sizes (top row $=20 \%$ most anterior/posterior ORs, $n=70$; middle row

$014=27.5 \%$ most anterior/posterior ORs, $\mathrm{n}=97$; bottom row $=35 \%$ most anterior $/$ posterior ORs,

$015 \mathrm{n}=123)$ used as conditions for identifying significantly different residues as compared to 
016 Class II dorsal OB ORs. (B) Phylogenetic trees for anterior sets of Class II ventral OB ORs

017 (blue, left), posterior sets of Class II ventral OB ORs (red, middle), and combined sets of

018 ventral anterior and posterior Class II OB ORs (right). Sets of different sizes (top row $=20 \%$

019 most anterior/posterior ORs, $\mathrm{n}=92$; middle row $=27.5 \%$ most anterior/posterior ORs, $\mathrm{n}=$ 020127 ; bottom row $=35 \%$ most anterior/posterior ORs, $n=162)$ used as conditions for

021 identifying significantly different residues as compared to Class II dorsal OB ORs. (C)

022 Distributions of dorsal and ventral anterior and posterior ORs by OR family as classified in

023 Olender et al. BMC Evol. Bio. 2020 for dorsal OB ORs (left) and ventral OB ORs (middle).

024 Distribution of all Class II dorsal and ventral ORs by OR family is depicted in the top right. 


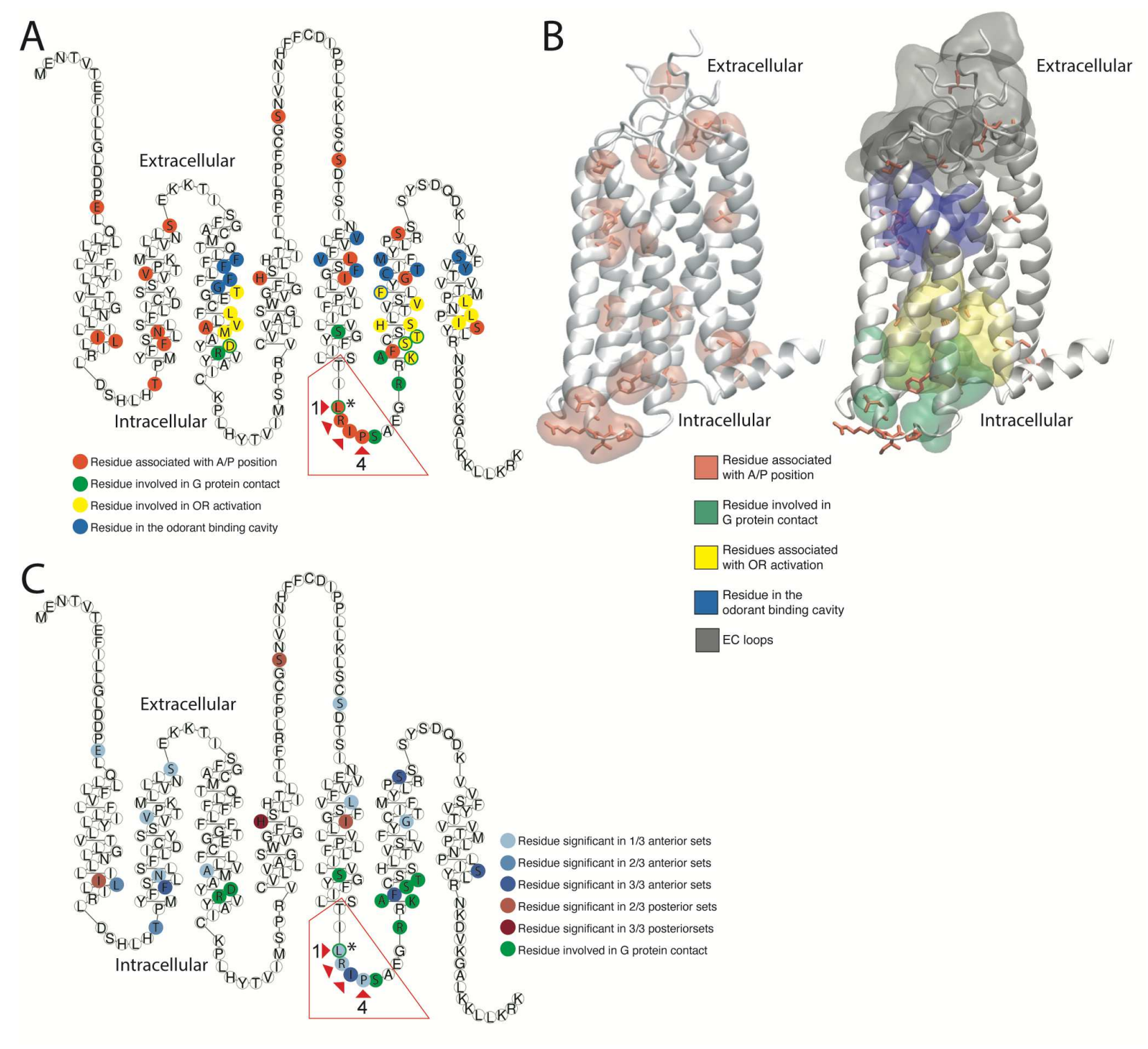

026 Fig. S8. Position of anterior and posterior associated residues within ventral Class II

027 ORs. (A) Snakeplot of the Class II OR consensus protein sequence with residues color coded

028 as being associated with glomerular AP position (orange), involved in G protein contact

029 (green), involved in the OR activation mechanism (yellow), and located in the odorant binding

.030 cavity (blue). Residues filled with one color and bordered in a different color were associated

031 with both categories. (B) Homology model of the mouse Class II consensus OR. Left,

032 residues associated with AP positions (orange) are depicted in licorice with transparent

033 regions indicating residue surface. Right, transparent regions represent the surface of 
034 residues located in the extracellular (EC) loops (gray), located in the odorant binding cavity

035 (blue), associated with the activation mechanism (yellow) and associated with $\mathrm{G}$ protein

036 binding (green). (C) Snakeplot of the Class II OR consensus protein sequence with blue

037 shaded residues were identified as having significant different physicochemical properties for

038 ventral, anterior, Class II ORs compared to all ventral Class II ORs. Red shaded residues

039 were identified as having significantly different physicochemical properties for ventral,

040 posterior, Class II ORs compared to all ventral Class II ORs. Residues highlighted in green

041 indicate mammalian OR residues known to be involved in Class A GPCR activation through

042 contact with the $\mathrm{G}$ protein ${ }^{*}$ indicates the single residue which was identified as being both

043 associated with $\mathrm{G}$ protein contact and identified as significantly different for ventral, anterior,

044 Class II ORs). 


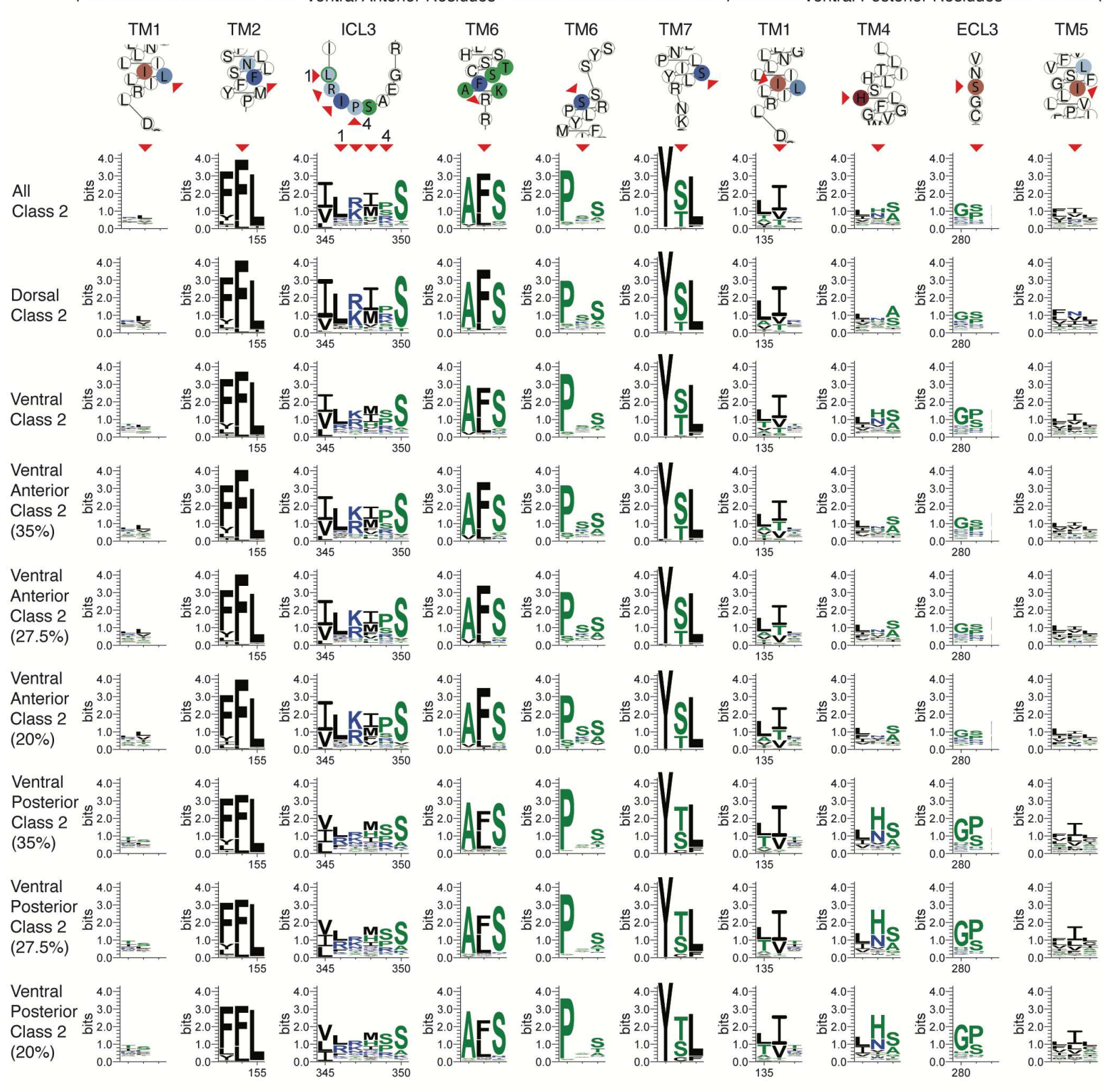

046 Fig. S9. Sequence logos for selected ventral Class II anterior and posterior residues.

047 Protein sequence logos for positions associated with ventral Class II anterior OR residues

048 (left six columns) and ventral Class II posterior OR residues (right four columns) depicting the

049 conservation of specific amino acid residues within different sets of sequences. Red arrows

050 indicate the specific residue within the Class II OR consensus snakeplot (fig. S8, A and C)

051 and the corresponding position in the sequence logo. 

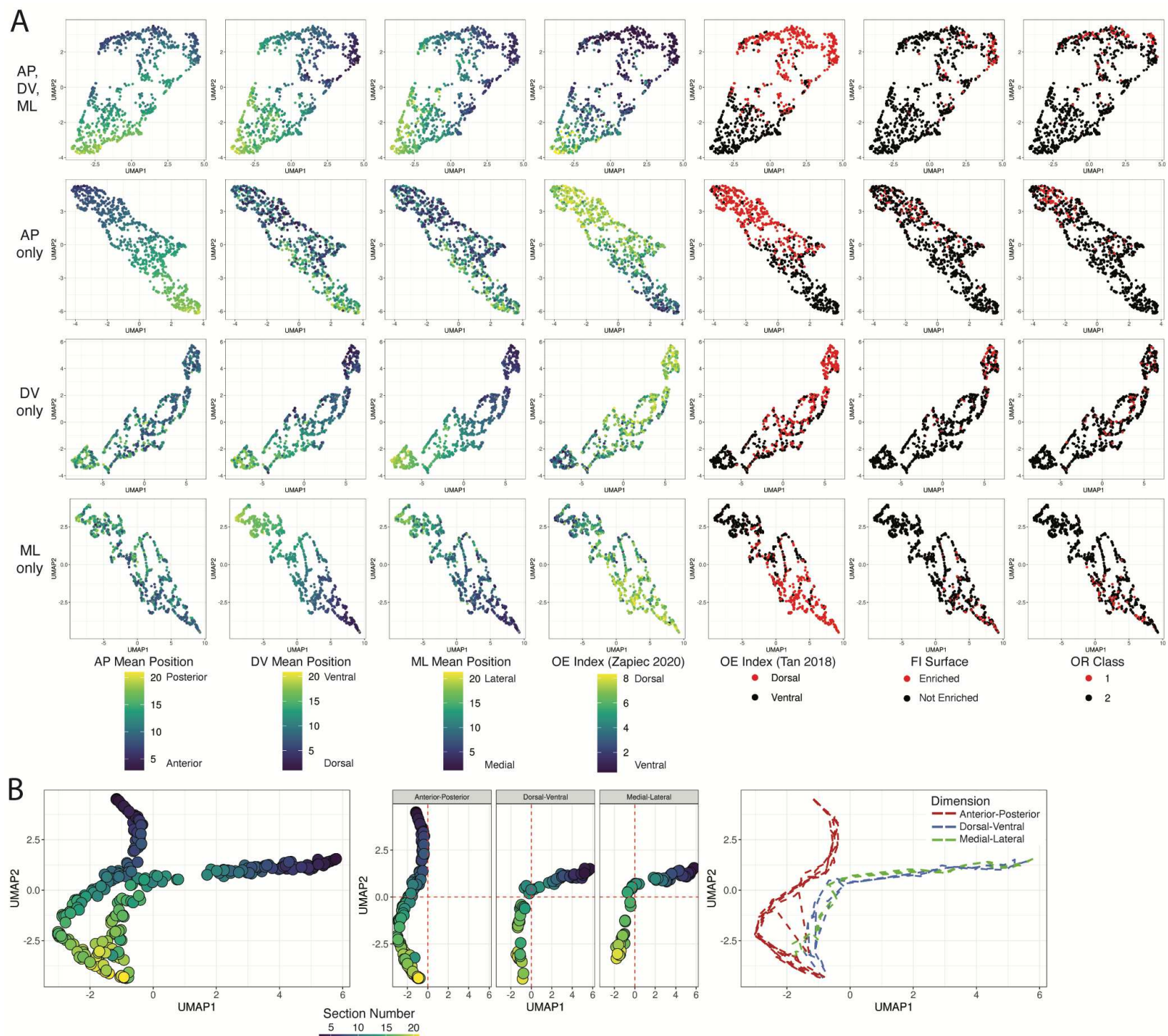

052

053 Fig. S10. Positional features are conserved when only mean position data is used. (A)

054 UMAP projections of OR populations constructed using the mean position from each replicate 055 from all dimensions (top row), AP replicates only (second row), DV replicates only (third row), 056 and $\mathrm{ML}$ replicates only (bottom row). AP, DV, and ML mean position reflects the calculated 057 average mean position from each dimension. OE Index (Zapiec 2020) refers to the OE index 058 established by Zapiec and Mombaerts Cell Reports 2020. OE Index (Tan 2018) refers to the 059 OE index established by Tan and Xie Chem. Senses 2018 with values less than 2 labeled as 060 Dorsal and equal or greater than 2 labeled as Ventral. FI Surface refers to the differential 
061 expression analysis calculated from dorsal and ventral OB samples in this paper. (B) UMAP 062 projection of captured OB spatial samples constructed using transcript abundances for the 063980 ORs and TAARs from all dimensions (left), separated by dimension (middle), and for the 064 order of section number by replicate from each dimension (right). 


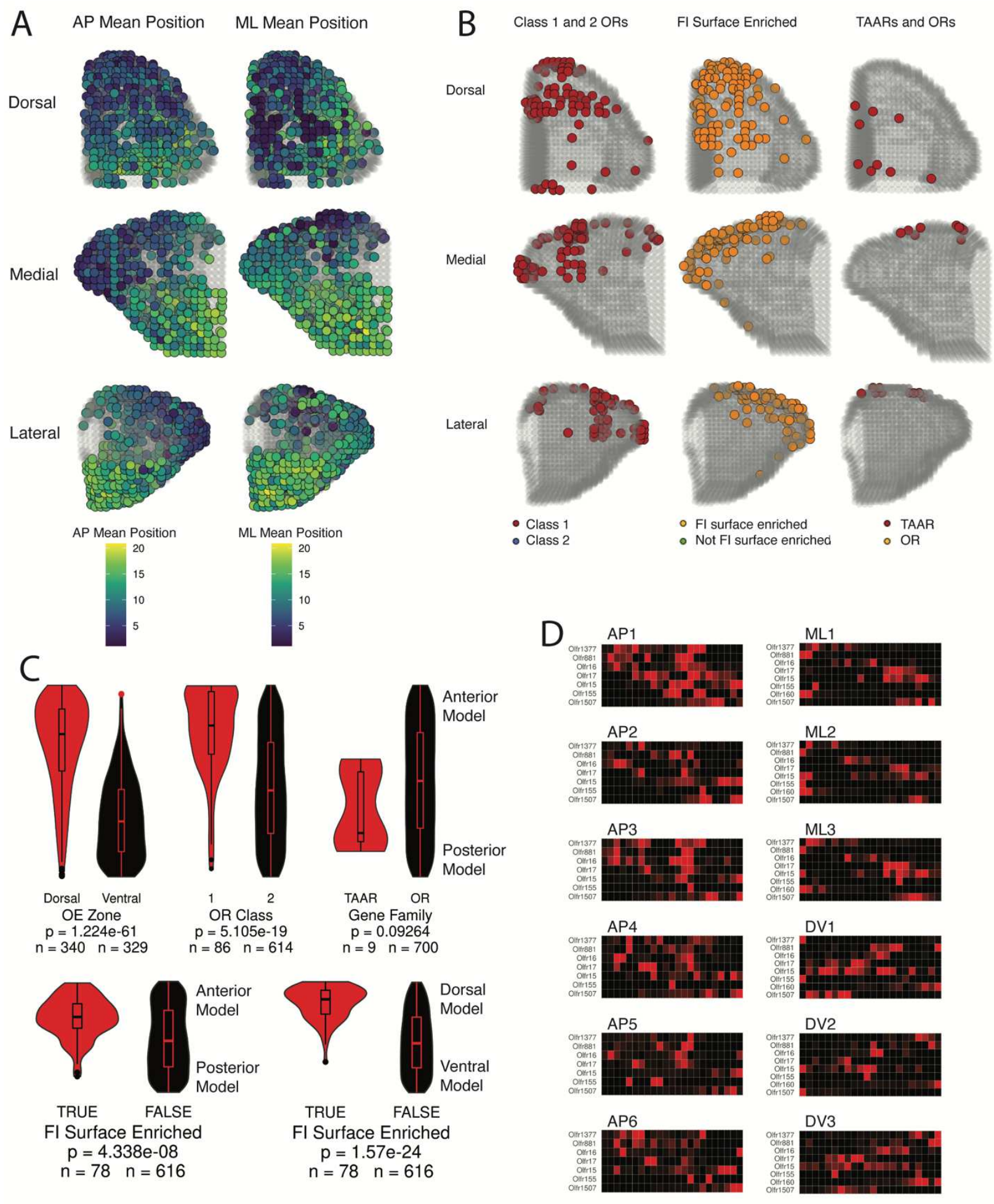

066 Fig. S11. A three-dimensional model for OR glomeruli positions from single-dimension

067 targeted sequencing data. (A) Three-dimensional predictions for all 709 ORs and TAARs

068 colored by AP mean position (left) and ML mean position (right). (B) Three-dimensional 
069 predictions for the 709 ORs and TAARs colored to show the distribution of Class I, functional

070 imaging surface enriched ORs, and TAARs without opposing features in order to better

071 demarcate positions. (C) Distribution of ranked mean model position (AP axis for top row and

072 bottom left and DV axis for bottom right) for the best probability voxel in each predicted

073 glomerulus for all 709 ORs and TAARs for OE zone, OR Class, gene family, and enrichment

074 in the functional imaging surface. Statistic is Mann-Whitney U-test. (D) Heatmaps for labeled

075 ORs from individual replicates along the AP (left), ML (top right), and DV (bottom right)

076 dimensions. 
A

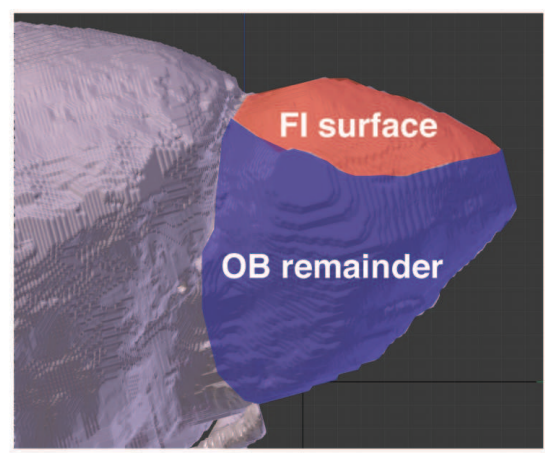

C
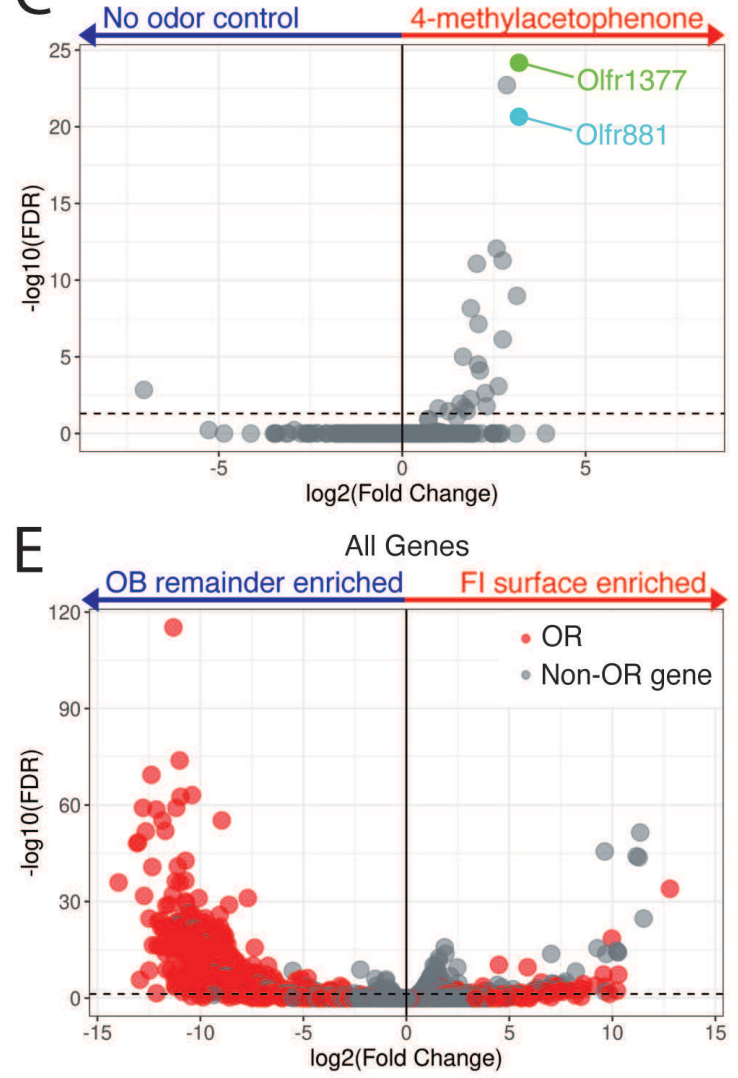

G

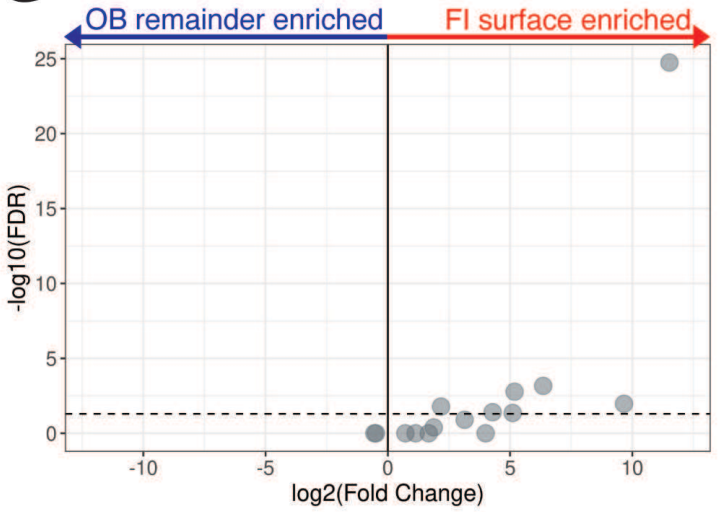

B

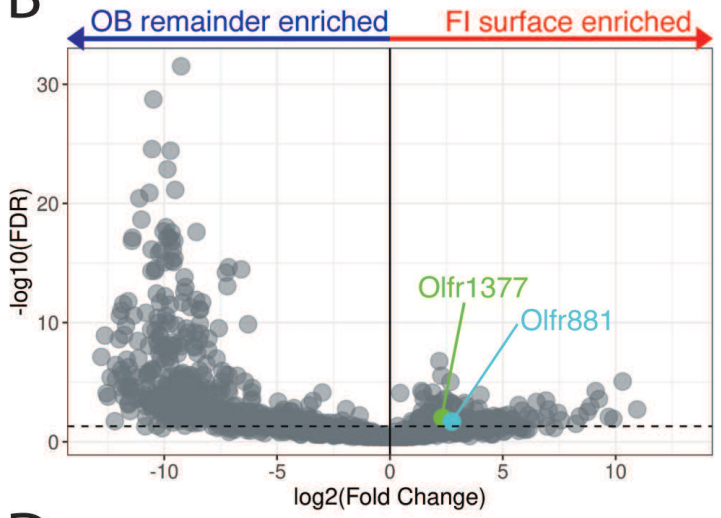

D

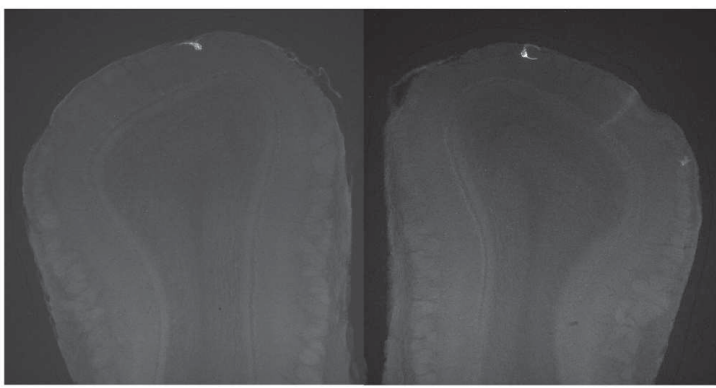

$\mathrm{F}$

F Only ORs

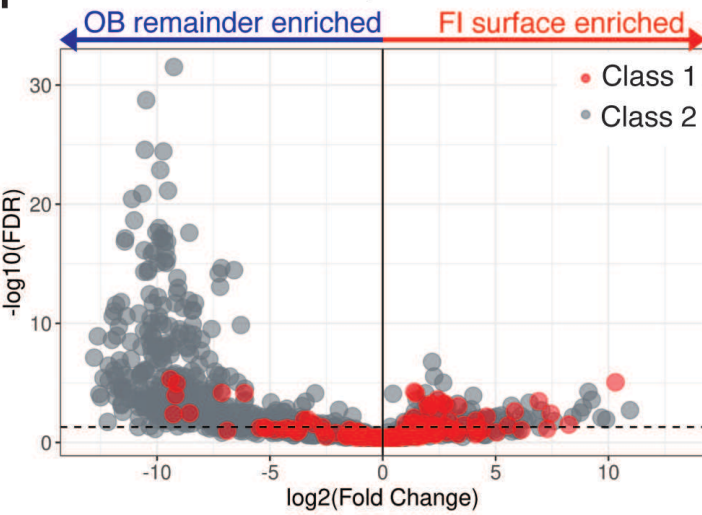

$\mathrm{H}$

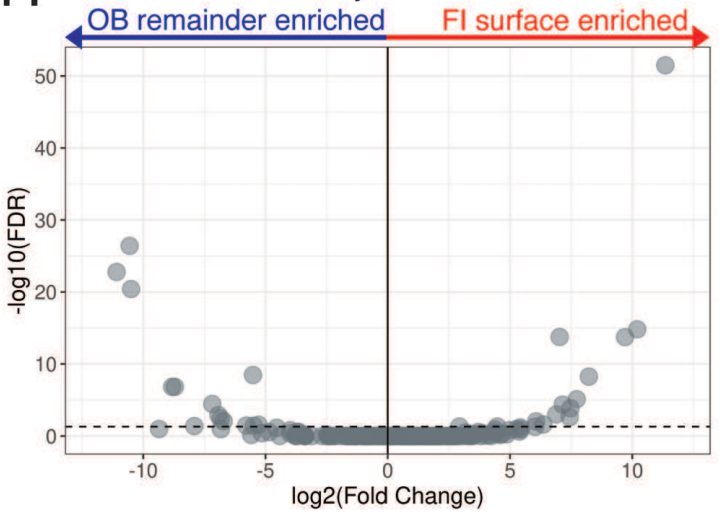


079 Fig. S12. Differential expression analysis for functional imaging surface samples. (A)

080 OB schematic showing approximation of functional imaging (FI) surface (red) and OB

081 remainder (blue) dissected for differential expression analysis. (B) Volcano plot comparing

082 expression of ORs in functional imaging surface and OB remainder samples. The FI surface

083 enriched ORs, Olfr1377 and Olfr881 are labeled in green and cyan, respectively. (C) Volcano

084 plot comparing expression of ORs in pS6-IP RNA-Seq stimulation with 1\% 4-

085 methylacetophenone. The highly ranked responding ORs, Olfr1377 and Olfr881 are labeled in

086 green and cyan, respectively. (D) Coronal sections from an Olfr881-IRES-mKate2 mouse

087 indicating a dorsal-central location of the mKate2 labeled ORs (E) Volcano plots for

088 differential expression from functional imaging samples and OB remainder samples for all

089 genes, ORs labeled in red. (F) Volcano plot for only ORs, FDR readjusted, Class I ORs

090 labeled in red. (G) Volcano plot for only TAARs, FDR readjusted. (H) Volcano plot for only

091 vomeronasal receptors (VMNRs), FDR readjusted. 


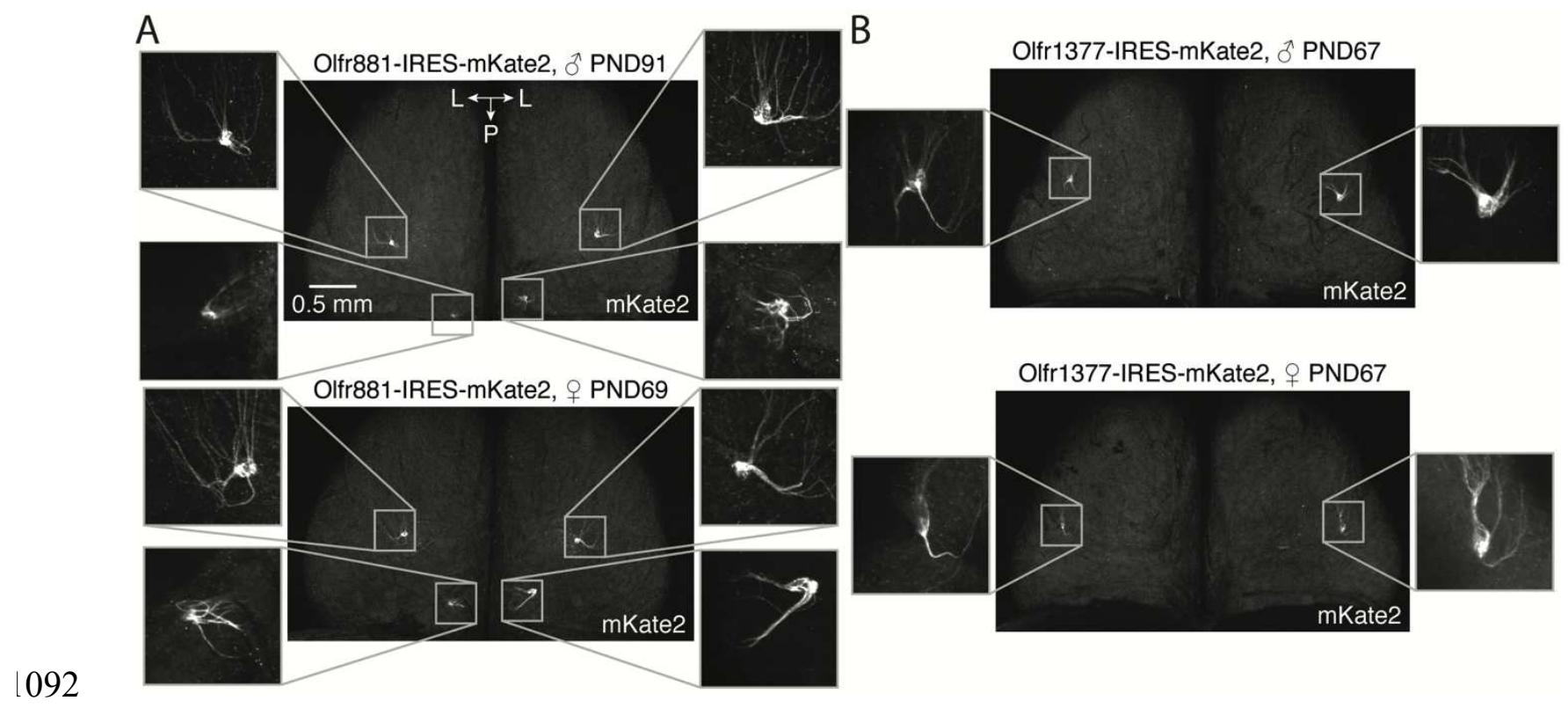

093 Fig. S13. Whole-mount confocal microscopy and glomerular phenotypes of Olfr1377-

094 IRES-mKate2 and OIfr881-IRES-mKate2 OBs. (A) Whole-mount maximum intensity

095 projections of the dorsal surface of Olfr881-IRES-mKate2 OBs from male and female mice

096 with inlays showing the mKate2-positive glomeruli. (B) Whole-mount maximum intensity

097 projections of the dorsal surface of Olfr1377-IRES-mKate2 OBs from male and female mice 098 with inlays showing the mKate2-positive glomeruli. 


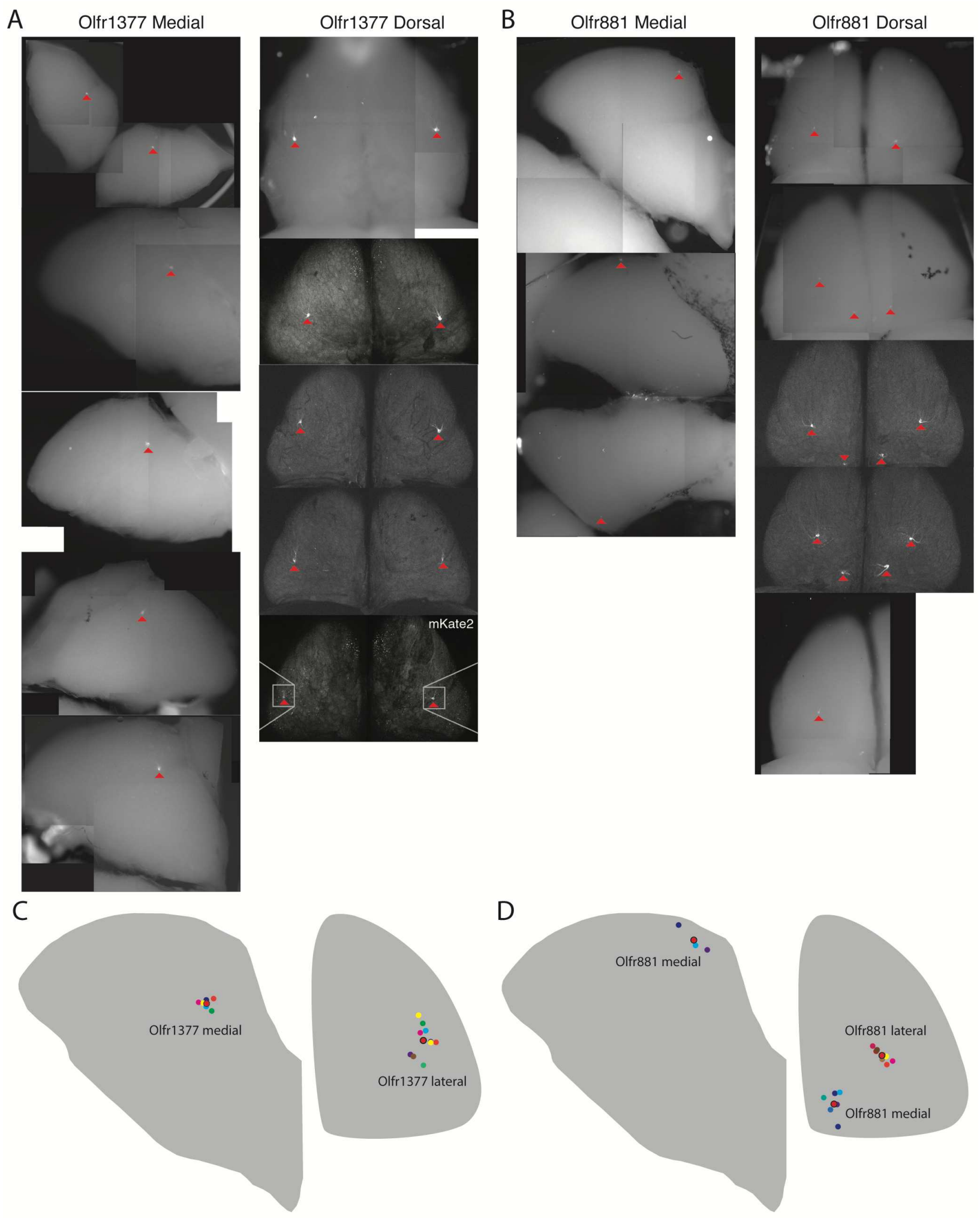

100 Fig. S14. Position of Olfr1377 and Olfr881 glomeruli. (A) Whole-mount stitched

101 epifluorescence images (together with whole-mount confocal maximal intensity projections 
102 from fig. S13) reconstructing the medial (left) and dorsal (right) faces of Olfr1377-IRES-

103 mKate2 mice OBs. Red arrows indicate glomerulus. (B) Same as (A) for Olfr881-IRES-

104 mKate2 mice. (C) Positions of Olfr1377 fluorescent glomeruli as recorded from the whole-

105 mount OB images. Red bordered circles indicate the mean position of the individual

106 replicates. (D) Same as (C) for Olfr881. 

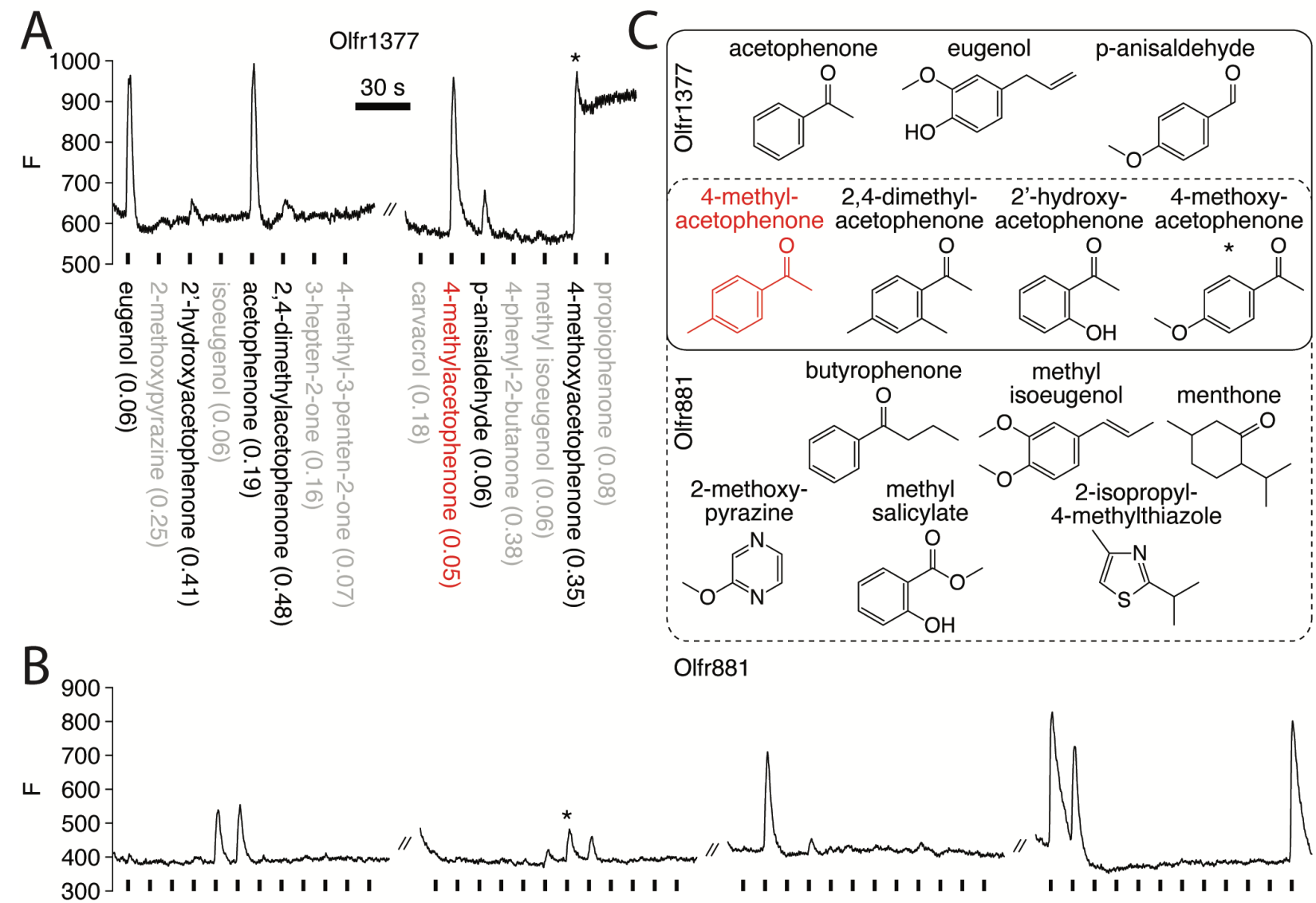

Olfr881
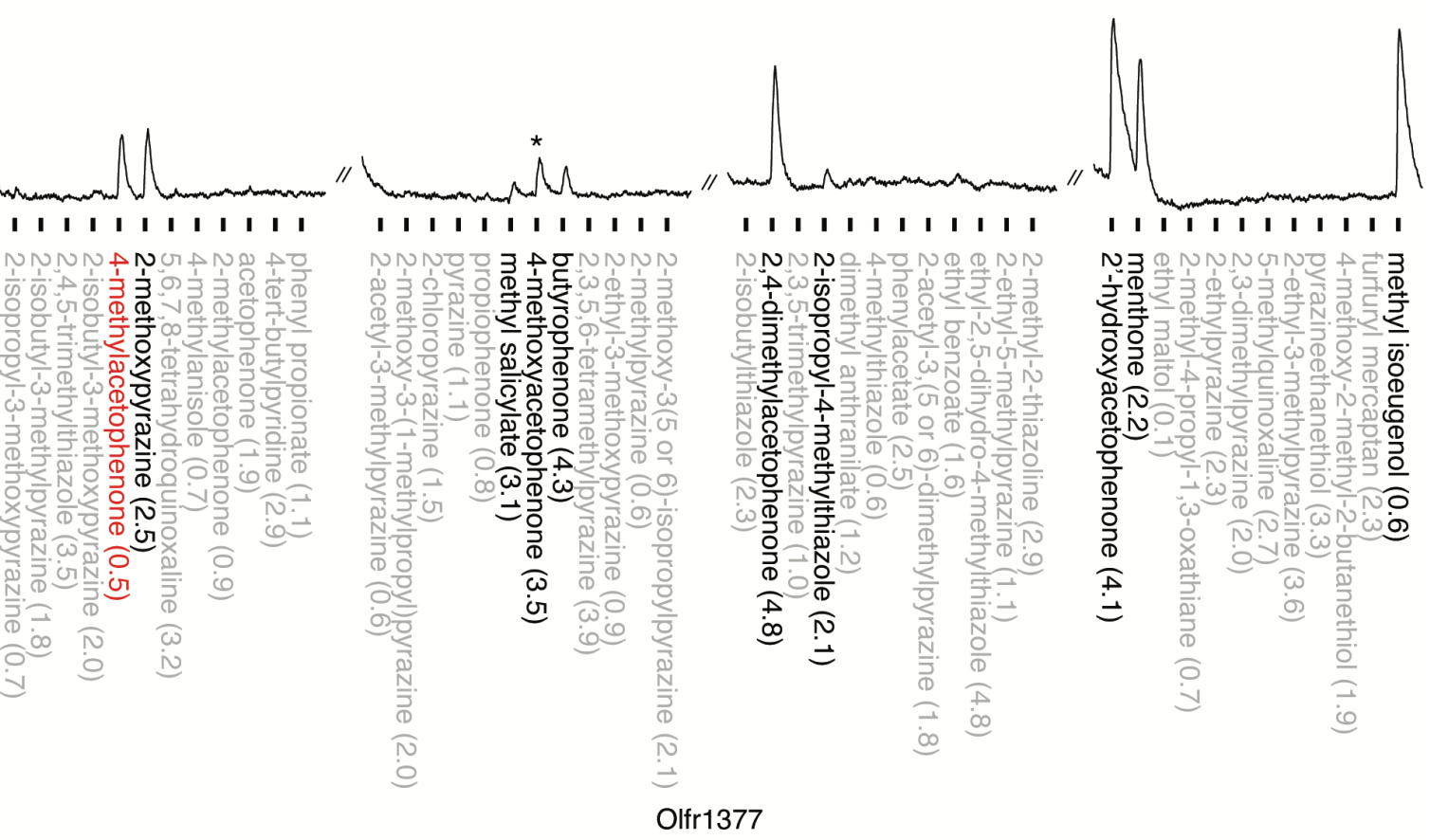

$D^{2200}$
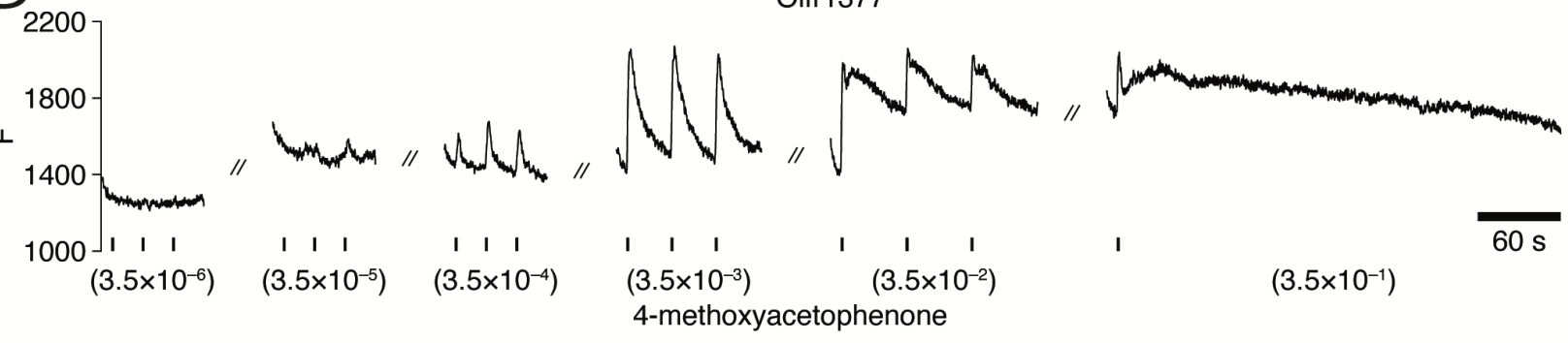

$\left(3.5 \times 10^{-1}\right)$

108 Fig. S15. In vivo two-photon GCaMP6s response kinetics and concentration- 
110 fluorescence of a mKate2-labeled Olfr1377 glomerulus during sequential presentation of

111 odorants in pseudorandom order to a compound heterozygous Olfr1377-IRES-mKate2; OMP-

112 IRES-tTA; tetO-GCaMP6s mouse. * marks long-term activation by 4-methoxyacetophenone.

113 Red text highlights response to 4-methylacetophenone. Odorants presented at an estimated

114 concentration on the order of $10^{-1} \mathrm{nM}$. (B) Similar to (A) for a mKate2-labeled Olfr881

115 glomerulus imaged in a compound heterozygous Olfr881-IRES-mKate2; OMP-IRES-tTA;

116 tetO-GCaMP6s mouse. Note the lack of long-term activation by 4-methoxyacetophenone.

117 Odorants presented at an estimated concentration on the order of $10^{\circ} \mathrm{nM}$. (C) Chemical

118 structures of a subset of ligands identified by functional imaging for Olfr1377 (solid box) and

119 Olfr881 (dashed box), including multiple overlapping cyclic ketone ligands. (D) Raw two-

120 photon GCaMP6s fluorescence of a mKate2-labeled Olfr1377 glomerulus during sequential

121 presentation of increasing concentrations of 4-methoxyacetophenone, revealing long-term

122 activation with $3.5 \times 10^{-2} \mathrm{nM}$ and higher concentrations. 
124 Table S1: Mice used for sequencing

125 Table S2: Sequencing read depth for each sample

126 Table S3: Probe coverage and enrichment statistics

127 Table S4: Target gene expression and normalization weights

128 Table S5: Dimensional mean positions for target genes

129 Table S6: Summary statistics for percent identity score-based groups of Class I, Class II

130 Dorsal OB ORs, and Class II Ventral OB ORs

131 Table S7: Residue identities of Class II OR consensus sequence and significance for all 132 tested sets of anterior/posterior ORs

133 Table S8: Three-dimensional model posterior medians

134 Table S9: Differential expression analysis for functional imaging surface and pS6-IP 4-

135 methylacetophenone studies

136 Table S10: Heterologous cell assay results 


\section{Supplementary Files}

This is a list of supplementary files associated with this preprint. Click to download.

- obmapsuptables.zip 